\title{
A SPATIO-TEMPORAL DESIGN PROBLEM FOR A DAMPED WAVE EQUATION*
}

\author{
FAUSTINO MAESTRE ${ }^{\dagger}$, ARNAUD MÜNCH $^{\ddagger}$, AND PABLO PEDREGAL $^{\dagger}$
}

\begin{abstract}
We analyze in this work a spatio-temporal optimal design problem governed by a linear damped one-dimensional wave equation. The problem consists of simultaneously seeking the spatio-temporal layout of two isotropic materials and the static position of the damping set in order to minimize a functional depending quadratically on the gradient of the state. The lack of classical solutions for this kind of nonlinear problem is well known. We examine a well-posed relaxation by using the representation of a two-dimensional divergence-free vector as a rotated gradient. We transform the original optimal design problem into a nonconvex vector variational problem. By means of gradient Young measures we compute an explicit form of the "constrained quasi convexification" of the cost density. Moreover, this quasi convexification is recovered by first order laminates which give the optimal distribution of materials and damping set at every point. Finally, we analyze the relaxed problem, and some numerical experiments are performed. The novelty here lies in the optimization with respect to two independent subdomains, and our contribution consists of understanding their mutual interaction.
\end{abstract}

Key words. optimal design, wave equation, relaxation, Young measure

AMS subject classifications. 35L05, 49J05, 65K10

DOI. $10.1137 / 07067965 \mathrm{X}$

1. Introduction - Problem statement. Let us consider the following damped wave equation posed in $(0, T) \times \Omega$ :

$$
\begin{cases}u_{t t}-\nabla_{x}\left(\left[\alpha \mathcal{X}_{\omega_{1}}+\beta\left(1-\mathcal{X}_{\omega_{1}}\right)\right] u_{x}\right)+d(x) \mathcal{X}_{\omega_{2}} u_{t}=0 & \text { in }(0, T) \times \Omega \\ u=0 & \text { on }(0, T) \times \partial \Omega \\ u(0, x)=u_{0}(x), u_{t}(0, x)=u_{1}(x) & \text { in } \Omega\end{cases}
$$

for any bounded interval $\Omega$ of $\mathbb{R}$ and any positive time $T$. $\mathcal{X}_{\omega_{1}}$ and $\mathcal{X}_{\omega_{2}}$ designate respectively the characteristic function of two subsets $\omega_{1} \subset \Omega \times(0, T)$ and $\omega_{2} \subset \Omega$, both of positive Lebesgue measure $\left|\omega_{1}\right|$ and $\left|\omega_{2}\right|$. We assume that $0<\alpha<\beta$ and that the damping potential $d \in L^{\infty}\left(\Omega ; \mathbb{R}^{+}\right)$is such that $d(x) \geq d>0$ for all $x \in \omega_{2}$. Finally, we assume that the initial data $\left(u_{0}, u_{1}\right)$ are in $H_{0}^{1}(\Omega) \times L^{2}(\Omega)$ and are independent of $\omega_{1}, \omega_{2}$, and $d$. System (1.1) is then well posed, and there exists a unique weak solution such that $u \in C\left([0, T] ; H_{0}^{1}(\Omega)\right) \cap C^{1}\left([0, T] ; L^{2}(\Omega)\right)$ (see $\left.[16]\right)$.

As is well known, system (1.1) models the stabilization of an elastic string made of two materials $\alpha$ and $\beta$ located on $\omega_{1}$ and $\left.((0, T) \times \Omega)\right) \backslash \omega_{1}$, respectively, by an internal dissipative mechanism located on $\omega_{2}$. The unknown $u(t, x)$ represents the transversal displacement of the string at the point $x$ and at time $t$, while $u_{0}$ and $u_{1}$ designate the initial position and velocity, respectively.

\footnotetext{
* Received by the editors January 9, 2007; accepted for publication (in revised form) May 18, 2007; published electronically October 17, 2007.

http://www.siam.org/journals/siap/68-1/67965.html

${ }^{\dagger}$ ETSI Industriales, Universidad de Castilla-La Mancha, 13071 Ciudad Real, Spain (Faustino. Maestre@uclm.es, Pablo.Pedregal@uclm.es). The research of the first author was supported by project MTM2004-07114 from Ministerio de Educación y Ciencia (Spain), by project PAI05-029 from JCCM (Castilla-La Mancha), and by Ph.D. grant 03/034 of JCCM. The research of the third author was supported by project MTM2004-07114 from Ministerio de Educación y Ciencia (Spain) and by project PAI05-029 from JCCM (Castilla-La Mancha).

${ }^{\ddagger}$ Corresponding author. UMR CNRS 6623, Université de Franche-Comte, 16 route de Gray, 25030 Besançon cedex, France (arnaud.munch@univ-fcomte.fr).
} 
Following similar works $[9,15]$, we address the very important question of determining the best space-time layout of materials $\alpha$ and $\beta$ in $\Omega \times(0, T)$ and the best space distribution of damping material in order to minimize some cost depending on the square of the gradient of the underlying state $u$. Precisely, introducing the functions $a_{\alpha}, a_{\beta} \in L^{\infty}\left((0, T) \times \Omega ; \mathbb{R}_{+}^{\star}\right)$ and

$$
a\left(t, x, \mathcal{X}_{\omega_{1}}\right)=\mathcal{X}_{\omega_{1}} a_{\alpha}(t, x)+\left(1-\mathcal{X}_{\omega_{1}}\right) a_{\beta}(t, x),
$$

we consider the following nonlinear optimal shape design problem:

$$
\inf _{\mathcal{X}_{\omega_{1}}, \mathcal{X}_{\omega_{2}}} I\left(\mathcal{X}_{\omega_{1}}, \mathcal{X}_{\omega_{2}}\right)=\int_{0}^{T} \int_{\Omega}\left(u_{t}^{2}+a\left(t, x, \mathcal{X}_{\omega_{1}}\right)\left|u_{x}\right|^{2}\right) d x d t
$$

subject to

$$
\left\{\begin{array}{l}
u \text { fulfills }(1.1), \\
\mathcal{X}_{\omega_{1}} \in L^{\infty}(\Omega \times(0, T) ;\{0,1\}), \quad \mathcal{X}_{\omega_{2}} \in L^{\infty}(\Omega ;\{0,1\}), \\
\int_{\Omega} \mathcal{X}_{\omega_{1}}(t, x) d x \leq L_{\alpha}|\Omega| \quad \forall t \in(0, T), \quad L_{\alpha} \in(0,1), \\
\int_{\Omega} \mathcal{X}_{\omega_{2}}(x) d x \leq L_{d}|\Omega|, \quad L_{d} \in(0,1) .
\end{array}\right.
$$

The constraint $(1.4)_{3}$ requires that for all $t \in(0, T)$ the volume fraction of the $\alpha$ material be lower than $L_{\alpha}$ given in $(0,1)$. The constraint $(1.4)_{4}$ requires that the volume fraction of the damping material be lower than $L_{d}$ given in $(0,1)$.

Optimal design problems in conductivity and elasticity have been extensively studied in the last decade from various perspectives (e.g., the homogenization approach $[1,24]$, shape derivative $[6,7]$, topological derivative [27], variational formulation $[5,26]$, simulation-oriented approaches [4, 12], etc). Under the hyperbolic laws, much less is known. A pioneer work in this direction is [18], where the author analyzes the hyperbolic G-closure for a similar optimal control problem (see also [17] for a general report on dynamic materials). On the other hand, an interesting analysis for optimal control problems under the wave equation in greater dimensions is described in [8], where the control is a time dependent coefficient. Let us also mention [3], where the authors examine time-harmonic solutions of the wave equation, prove a relaxation result for the corresponding design problem, and obtain existence of classical solutions for some particular cases. Finally, shape analysis for noncylindrical evolution problems is considered in [7] (and the references therein).

More recently, a one-dimensional (1-D) hyperbolic optimal control design problem with designs depending both on $x$ and $t$ has been addressed in [20]. This corresponds to the problem (P) with $\omega_{2}=\emptyset$ and a minimization with respect to $\omega_{1}$ only. A full relaxation of the associated problem is given and numerically justified if the gap $\beta-\alpha>0$ is large enough. On the other hand, the pure damping case (corresponding to $\omega_{1}=\emptyset$ and a minimization with respect to $\omega_{2}$ only) has been studied similarly in $[13,21,22]$. Once again, it appears that the well-posed character of the problem relies on the amplitude of the function $d$. In this work, we aim at mixing these two cases and minimize $I$ with respect to $\omega_{1}$ and $\omega_{2}$ simultaneously. In this respect, we derive and analyze a well-posed relaxation of $(\mathrm{P})$. The approach is based on an equivalent variational reformulation of the original problem as a nonconvex vector variational problem: following $[2,26]$, we transform our scalar problem with differential constraints into a vector variational problem with integral constraints (where the state 
equation is implicit in the new cost function). It is well known that the nonexistence of optimal solutions for vector variational problems is related to the lack of quasi convexity of the cost functional $I$ (see [11]). Therefore, by using gradient Young measures as generalized solutions of variational problems, we compute an explicit relaxation of the original problem in the form of a relaxed (quasi-convexified) variational problem.

To the knowledge of the authors, this work is the first considering a bidesign problem. Our contribution consists, first, of adapting relaxation techniques in this case, and then, of studying the interaction between the two optimal designs $\omega_{1}$ and $\omega_{2}$.

The rest of the paper is organized as follows. In section 2, we describe in detail the equivalent variational reformulation (denoted by (VP)) as well as a general relaxation result when integrands are not continuous and may take on infinite values abruptly. Section 3 presents the computation of the constrained quasi convexification of the underlying integrand of (VP). The first part is concerned with the computation of a lower bound - the constrained polyconvexification - by using in a fundamental way the weak continuity of the determinant. The second part is concerned with the search for laminates furnishing the precise value of the lower bound in an attempt to show equality of the three convex hulls (poly-, quasi-, and rank one convex hulls). This provides the well-posed relaxation (RP) stated in Theorem 3.4. In addition, the optimal Young measure permits us to describe precisely the optimal microstructure (see Theorem 3.5). Section 4 is devoted to the analysis of the relaxed formulation. In section 5, we present some numerical experiments which justify the introduction of the relaxed formulation (RP) and present a simple penalization technique to obtain some elements of a minimizing sequence for $(\mathrm{P})$ from the relaxed optimal solution of $(\mathrm{RP})$.

2. Variational reformulation and relaxation. In order to apply suitable results of calculus of variations $[11,25]$, we first reformulate the problem $(\mathrm{P})$ into a classical vector variational one. To this end, following $[2,19,26]$, we use a characterization of divergence-free vector fields. Precisely, since the subset $\omega_{2}$ is time independent, the state equation of system (1.1) can be written as

$$
\operatorname{div}\left(u_{t}+d(x) \mathcal{X}_{\omega_{2}} u,-\left[\alpha \mathcal{X}_{\omega_{1}}+\beta\left(1-\mathcal{X}_{\omega_{1}}\right)\right] u_{x}\right)=0
$$

where the operator div is defined as div $=\left(\partial_{t}, \nabla_{x}\right)$. Then, under the hypothesis of simple-connectedness of $\Omega$ and from the characterization of the 2-D divergencefree vector fields (see, for instance, [14], Chapter I), there exists a potential $v \in$ $H^{1}(\Omega \times(0, T))$ such that the above formula is equivalent to the pointwise constraint

$$
\left(\begin{array}{c}
u_{t} \\
-\left(\alpha \mathcal{X}_{\omega_{1}}+\beta\left(1-\mathcal{X}_{\omega_{1}}\right)\right) u_{x}
\end{array}\right)-R \nabla v=-d(x) \mathcal{X}_{\omega_{2}} \bar{u}
$$

where

$$
\bar{u}=\left(\begin{array}{l}
u \\
0
\end{array}\right), \quad \nabla v=\left(\begin{array}{l}
v_{t} \\
v_{x}
\end{array}\right), \quad R=\left(\begin{array}{cc}
0 & -1 \\
1 & 0
\end{array}\right)
$$

$R$ is the counterclockwise $\pi / 2$-rotation in the $(x, t)$-plane. We then introduce the vector field $U=(u, v) \in\left(H^{1}(\Omega \times(0, T))\right)^{2}$ and the manifolds $\Lambda_{\gamma, \lambda}$ as follows:

$$
\Lambda_{\gamma, \lambda}=\left\{A \in M^{2 \times 2}: M_{-\gamma} A^{(1)}-R A^{(2)}=\lambda e_{1}\right\}, \quad \gamma=\alpha, \beta, \text { and } \lambda \in \mathbb{R}
$$


where $A^{(i)}, i=1,2$, stands for the $i$ th row of the matrix and

$$
M_{-\gamma}=\left(\begin{array}{cc}
1 & 0 \\
0 & -\gamma
\end{array}\right), \quad e_{1}=\left(\begin{array}{l}
1 \\
0
\end{array}\right)
$$

It is clear that we can identify the design variable $\left(\mathcal{X}_{\omega_{1}}, \mathcal{X}_{\omega_{2}}\right)$ with the vector field $U=$ $(u, v)$; conversely, a pair $U=(u, v)$ which verifies $(2.2)$ determines characteristic functions $\left(\mathcal{X}_{\omega_{1}}, \mathcal{X}_{\omega_{2}}\right)$, so that we can consider the new design variables $U=(u, v)$, where $U: \mathbb{R}^{2} \rightarrow \mathbb{R}^{2}$ and $\nabla U(t, x) \in \mathbb{R}^{2 \times 2}$. Then, for any $2 \times 2$ matrix $A=\left(a_{i j}\right)_{(1 \leq i, j \leq 2)}$, we consider the following three functions:

$$
\begin{gathered}
W(t, x, U, A)= \begin{cases}a_{11}^{2}+a_{\alpha}(t, x) a_{12}^{2} & \text { if } A \in \Lambda_{\alpha, 0} \cup \Lambda_{\alpha,-d(x) U^{(1)}}, \quad \backslash\left(\Lambda_{\alpha, 0} \cup \Lambda_{\alpha,-d(x) U^{(1)}}\right), \\
a_{11}^{2}+a_{\beta}(t, x) a_{12}^{2} & \text { if } A \in\left(\Lambda_{\beta, 0} \cup \Lambda_{\beta,-d(x) U^{(1)}}\right) \\
+\infty & \text { else, }\end{cases} \\
V_{\alpha}(t, x, U, A)= \begin{cases}1 & \text { if } A \in \Lambda_{\alpha, 0} \cup \Lambda_{\alpha,-d(x) U^{(1)}}, \\
0 & \text { if } A \in\left(\Lambda_{\beta, 0} \cup \Lambda_{\beta,-d(x) U^{(1)}}\right) \backslash\left(\Lambda_{\alpha, 0} \cup \Lambda_{\alpha,-d(x) U^{(1)}}\right), \\
+\infty & \text { else, }\end{cases} \\
V_{d}(t, x, U, A)= \begin{cases}1 & \text { if } A \in\left(\Lambda_{\beta,-d(x) U^{(1)}} \cup \Lambda_{\alpha,-d(x) U^{(1)}}\right), \\
0 & \text { if } A \in\left(\Lambda_{\beta, 0} \cup \Lambda_{\alpha, 0}\right) \backslash\left(\Lambda_{\beta,-d(x) U^{(1)}} \cup \Lambda_{\alpha,-d(x) U^{(1)}}\right), \\
+\infty & \text { else. }\end{cases}
\end{gathered}
$$

Then, noting that

$$
\left\{x \in \Omega, \mathcal{X}_{\omega_{1}}(x, t)=1\right\}=\left\{x \in \Omega, V_{\alpha}(t, x, U, \nabla U)=1\right\} \quad \forall t \in(0, T)
$$

and

$$
\left\{x \in \Omega, \mathcal{X}_{\omega_{2}}(x)=1\right\}=\left\{x \in \Omega, V_{d}(t, x, U, \nabla U)=1 \quad \forall t \in(0, T)\right\},
$$

the optimization problem $(\mathrm{P})$ is equivalent to the following vector variational problem:

$$
\text { (VP) } m=\inf _{U} \int_{0}^{T} \int_{\Omega} W(t, x, U(t, x), \nabla U(t, x)) d x d t
$$

subject to

$$
\left\{\begin{array}{l}
U=\left(U^{(1)}, U^{(2)}\right) \in H^{1}((0, T) \times \Omega)^{2}, \\
U^{(1)}(0, x)=u_{0}(x), U_{t}^{(1)}(0, x)=u_{1}(x) \quad \text { in } \Omega, \\
U^{(1)}=0 \text { in }(0, T) \times \partial \Omega, \\
\int_{\Omega} V_{\alpha}(t, x, U(t, x), \nabla U(t, x)) d x \leq L_{\alpha}|\Omega| \quad \forall t \in[0, T], \\
\int_{\Omega} V_{d}(t, x, U(t, x), \nabla U(t, x)) \times V_{d}(0, x, U(0, x), \nabla U(0, x)) d x \leq L_{d}|\Omega| \quad \forall t \in[0, T] .
\end{array}\right.
$$

Copyright (c) by SIAM. Unauthorized reproduction of this article is prohibited. 
Therefore, this procedure transforms the scalar dynamical problem $(\mathrm{P})$, with differentiable, integrable, and pointwise constraints, into a nonconvex vector variational problem (VP) with only pointwise and integral constraints.

We are now going to analyze the nonconvex vector problem (VP) by seeking its relaxation. We use Young measures (see [25]) as a main tool in the computation of the suitable density for the relaxed problem. Let us recall the following definition.

DEFINITION 2.1. The constrained quasi convexification of the functional $W$ is defined as

$$
C Q W(t, x, U, A, s, r)=\inf _{\nu}\left\{\int_{M^{2 \times 2}} W(t, x, U, A) d \nu(A): \nu \in \mathcal{A}\right\},
$$

where

$$
\begin{gathered}
\mathcal{A}=\left\{\nu: \nu \text { is a homogeneous } H^{1}\right. \text {-Young measure, } \\
F=\int_{M^{2 \times 2}} A d \nu(A), \int_{M^{2 \times 2}} V_{\alpha}(t, x, U, A) d \nu(A)=s, \\
\left.\int_{M^{2 \times 2}} V_{d}(t, x, U, A) d \nu(A)=r \quad \forall t \in[0, T]\right\} .
\end{gathered}
$$

We then introduce the following minimization problem:

$$
(\mathrm{RP}) \quad \bar{m}=\inf _{(U, s, r)} \int_{0}^{T} \int_{\Omega} C Q W(t, x, U(t, x), \nabla U(t, x), s(t, x), r(x)) d x d t
$$

subject to

$$
\left\{\begin{array}{l}
U=\left(U^{(1)}, U^{(2)}\right) \in H^{1}((0, T) \times \Omega)^{2}, \\
U^{(1)}(0, x)=u_{0}(x), U_{t}^{(1)}(0, x)=u_{1}(x) \quad \text { in } \quad \Omega, \\
U^{(1)}=0, \quad \text { in } \quad(0, T) \times \partial \Omega, \\
0 \leq s(t, x) \leq 1, \quad \int_{\Omega} s(t, x) d x \leq L_{\alpha}|\Omega| \quad \forall t \in[0, T], \\
0 \leq r(x) \leq 1, \quad \int_{\Omega} r(x) d x \leq L_{d}|\Omega| .
\end{array}\right.
$$

The functions $s$ and $r$ denote the pointwise volume fraction associated with the $\alpha$ material and the damping set, respectively.

Then, the following relaxation result (initially obtained in the elliptic case in $[2,26])$ can be proved: (RP) is a full relaxation of (VP) in the sense of the following theorem.

TheOREM 2.2. Assume that the initial data of system (1.1) have the regularity

$$
\left(u_{0}, u_{1}\right) \in\left(H^{2}(\Omega) \cap H_{0}^{1}(\Omega)\right) \times H_{0}^{1}(\Omega) .
$$

Then, problem (RP) is well posed and the following equality holds:

$$
m=\bar{m} \quad \text { (i.e., } \inf (\mathrm{VP})=\min (\mathrm{RP})) .
$$

Moreover, the minimum $(U, s, r)$ codifies (in the sense of Young measures) the optimal microstructures of the original optimal design problem.

Copyright (c) by SIAM. Unauthorized reproduction of this article is prohibited. 
Remark 2.3. In order to represent the limit of the cost function $I$ associated with a minimizing sequence, say $\left\{\mathcal{X}_{\omega_{1}, j}, \mathcal{X}_{\omega_{2}, j}\right\}_{j}$, through its associated Young measure, we need equi-integrability for the sequence $\left|u_{t, j}\right|^{2}+a\left(t, x, \mathcal{X}_{\omega_{1}, j}\right)\left|\nabla u_{j}\right|^{2}$ (see [25]). Equation (2.13) is a sufficient condition to get this equi-integrability. We refer to $[22,23]$ for the details.

Therefore, Theorem 2.2 reduces the determination of a relaxed formulation to the computation of the constrained quasi convexification $C Q W$ associated with $W$.

3. Constrained quasi convexification. In this section, we solve the optimization problem (2.9), leading for all $(U, F, s, r)$ to the value of $C Q W(t, x, U, F, s, r)$. The main difficulty is that we do not know explicitly the set of the admissible measures $\mathcal{A}$ defined in (2.10). We then follow the same strategy as in [26]. Consider two classes of a family of probability measures $\mathcal{A}_{\star}, \mathcal{A}^{\star}$ such that

$$
\mathcal{A}_{\star} \subset \mathcal{A} \subset \mathcal{A}^{\star} .
$$

We first calculate the minimum over the greater class of probability measures $\mathcal{A}^{\star}$, and then we check that the optimal value is attained by at least one measure over the narrower class $\mathcal{A}_{\star}$. This fact tells us that the optimal value so achieved is the same in $\mathcal{A}$, and hence we will have in fact computed the exact value $C Q W(t, x, U, F, s, r)$.

Following [26], we choose $\mathcal{A}^{\star}$ as the set of polyconvex measures, which are not necessarily gradient Young measures, and therefore obtain a lower bound (the constrained polyconvexification). The main property of these measures is that they commute with the determinant. This constraint can be imposed in a more-or-less manageable way. We also choose $\mathcal{A}_{*}$ as the class of laminates which is a subclass of the gradient Young measures. By working with this class, we would get an upper bound (the constrained rank one convexification).

In what follows, in order to simplify the expression, we note $\Lambda_{\gamma, 1}$ for $\Lambda_{\gamma,-d(x) U^{(1)}}$.

3.1. Lower bound: Polyconvexification. We compute the constrained polyconvexification defined as follows.

DeFinition 3.1. The constrained polyconvexification $C P W$ of the functional $W$ is given by the following minimization problem:

$$
C P W(U, F, s, r)=\min _{\nu}\left\{\int_{M^{2 \times 2}} W(U, A) d \nu(A): \nu \in \mathcal{A}^{\star}\right\},
$$

where

$$
\begin{aligned}
& \mathcal{A}^{\star}(F, s, r)=\{\nu: \nu \text { is a homogeneous Young measure, } \\
& \nu \text { commutes with the determinant, } \\
& F=\int_{M^{2 \times 2}} A d \nu(A), \\
& \left.s=\int_{M^{2 \times 2}} V_{\alpha}(U, A) d \nu(A), \quad r=\int_{M^{2 \times 2}} V_{d}(U, A) d \nu(A)\right\} .
\end{aligned}
$$

In this respect, we exploit that $\nu$ belongs to the class $\mathcal{A}^{\star}$. First, from the volume constraints $(3.2)_{4}$, the measure $\nu$ has the following decomposition:

$$
\nu=s\left(r \nu_{\alpha, 1}+(1-r) \nu_{\alpha, 0}\right)+(1-s)\left(r \nu_{\beta, 1}+(1-r) \nu_{\beta, 0}\right)
$$


with $\operatorname{supp}\left(\nu_{\gamma, \lambda}\right) \subset \Lambda_{\gamma, \lambda}, \gamma=\alpha, \beta, \lambda=0,1$. Therefore, if we introduce

$$
F^{\gamma, \lambda}=\int_{\Lambda_{\gamma, \lambda}} A d \nu_{\gamma, \lambda}, \quad \gamma=\alpha, \beta, \lambda=0,1
$$

then the first moment constraint $(3.2)_{3}$ leads to the following expression:

$$
F=s\left(r F^{\alpha, 1}+(1-r) F^{\alpha, 0}\right)+(1-s)\left(r F^{\beta, 1}+(1-r) F^{\beta, 0}\right) .
$$

Now, from the property $F^{\gamma, \lambda} \in \Lambda_{\gamma, \lambda}$, we have, for $\gamma=\alpha, \beta$,

$$
\left\{\begin{array} { l } 
{ F _ { 1 1 } ^ { \gamma , 0 } + F _ { 2 2 } ^ { \gamma , 0 } = 0 , } \\
{ - F _ { 2 1 } ^ { \gamma , 0 } - \gamma F _ { 1 2 } ^ { \gamma , 0 } = 0 , }
\end{array} \quad \text { and } \quad \left\{\begin{array}{l}
F_{11}^{\gamma, 1}+F_{22}^{\gamma, 1}=\lambda, \\
-F_{21}^{\gamma, 1}-\gamma F_{12}^{\gamma, 1}=0 .
\end{array}\right.\right.
$$

Substituting (3.5) into the system (3.4), we obtain a noncompatible system on $F^{\gamma, \lambda}$ unless the condition

$$
F_{11}+F_{22}=r \lambda
$$

holds. Assuming henceforth this compatibility condition, (3.4)-(3.6) lead to

$$
\left\{\begin{array}{l}
F_{11}^{\alpha, 1}=c_{1}, \quad F_{11}^{\alpha, 0}=c_{2}, \quad F_{11}^{\beta, 0}=c_{3}, \quad F_{12}^{\alpha, 1}=c_{4}, \quad F_{12}^{\beta, 1}=c_{5} \\
F_{11}^{\beta, 1}=\frac{F_{11}-r s c_{1}-s(1-r) c_{2}-(1-s)(1-r) c_{3}}{(1-s) r} \\
F_{12}^{\alpha, 0}=\frac{F_{21}+\beta F_{12}-(\beta-\alpha) r s c_{4}}{(1-r) s(\beta-\alpha)} \equiv f_{4}\left(c_{4}\right) \\
F_{12}^{\beta, 0}=\frac{-F_{21}-\alpha F_{12}-(\beta-\alpha) r(1-s) c_{5}}{(1-r)(1-s)(\beta-\alpha)} \equiv f_{5}\left(c_{5}\right)
\end{array}\right.
$$

where $c_{i} \in \mathbb{R}, i=1, \ldots, 5$, are parameters.

On the other hand, if we take a matrix $A=\left(a_{i j}\right)_{(1 \leq i, j \leq 2)} \in \Lambda_{\gamma, \lambda}$ with $\gamma=\alpha, \beta$ and $\lambda=0,1$, then the equality

$$
\operatorname{det} A=-A^{(1)} M_{-\gamma} A^{(1)}-\lambda A^{(1)} e_{1}
$$

and the constraint on the commutation yield

$$
\begin{aligned}
\operatorname{det} F= & \int_{M^{2 \times 2}} \operatorname{det} A d \nu(A) \\
=-S_{1}+\lambda r\left(s F_{11}^{\alpha, 1}+\right. & \left.(1-s) F_{11}^{\beta, 1}\right)+\alpha s\left(r S_{\alpha, 1}+(1-r) S_{\alpha, 0}\right) \\
& +\beta(1-s)\left(r S_{\beta, 1}+(1-r) S_{\beta, 0}\right),
\end{aligned}
$$

where

$$
S_{\gamma, \lambda}=\int_{\Lambda_{\gamma, \lambda}} a_{12}^{2} d \nu_{\gamma, \lambda}(A), \quad \gamma=\alpha, \beta, \lambda=1,0, \quad S_{1}=\int_{M^{2 \times 2}} a_{11}^{2} d \nu(A) .
$$

Similarly, the cost function can be written as (3.10)

$\int_{M^{2 \times 2}} W(U, A) d \nu(A)=S_{1}+a_{\alpha} s\left(r S_{\alpha, 1}+(1-r) S_{\alpha, 0}\right)+a_{\beta}(1-s)\left(r S_{\beta, 1}+(1-r) S_{\beta, 0}\right)$. 
Finally, using Jensen's inequality, we obtain

$$
S_{\gamma, \lambda}=\int_{\Lambda_{\gamma, \lambda}} a_{12}^{2} d \nu_{\gamma, \lambda} \geq\left|\int_{\Lambda_{\gamma, \lambda}} a_{12} d \nu_{\gamma, \lambda}\right|^{2}=\left|F_{12}^{\gamma, \lambda}\right|^{2}
$$

and

$$
S_{1} \geq\left|\int_{M^{2 \times 2}} a_{11} d \nu(A)\right|^{2}=\left|F_{11}\right|^{2} .
$$

As a conclusion, from (3.8), (3.10), (3.11), the polyconvexification problem (3.1) is reduced to the following mathematical programming problem:

$$
\min _{\left(S_{1}, S_{\gamma, \lambda}, c_{i}\right)} S_{1}+a_{\alpha} s\left(r S_{\alpha, 1}+(1-r) S_{\alpha, 0}\right)+a_{\beta}(1-s)\left(r S_{\beta, 1}+(1-r) S_{\beta, 0}\right)
$$

subject to

$$
\left\{\begin{aligned}
& \operatorname{det} F=\lambda r\left(s F_{11}^{\alpha, 1}+\right.\left.(1-s) F_{11}^{\beta, 1}\right)-S_{1} \\
&+\alpha s\left(r S_{\alpha, 1}+(1-r) S_{\alpha, 0}\right)+\beta(1-s)\left(r S_{\beta, 1}+(1-r) S_{\beta, 0}\right), \\
& S_{\gamma, \lambda} \geq\left(F_{12}^{\gamma, \lambda}\right)^{2}, \quad \gamma=\alpha, \beta, \quad \lambda=0,1 ; \quad S_{1} \geq\left(F_{11}\right)^{2}
\end{aligned}\right.
$$

The resolution of this problem leads to the following expression of $C P W$.

Proposition 3.2. The polyconvexification (3.1) is explicitly given by

$$
C P W(U, F, s, r)= \begin{cases}\left|F_{11}\right|^{2}+\frac{a_{\alpha}}{s(\beta-\alpha)^{2}}\left|\beta F_{12}+F_{21}\right|^{2} & \\ +\frac{a_{\beta}}{(1-s)(\beta-\alpha)^{2}}\left|\alpha F_{12}+F_{21}\right|^{2} & \text { if } \psi(F, s, r)=0, \\ +\infty & \text { else, }\end{cases}
$$

where

$$
\begin{aligned}
\psi(F, s, r)=-\operatorname{det} F-\left|F_{11}\right|^{2}+\lambda r F_{11} & +\frac{\alpha}{s(\beta-\alpha)^{2}}\left|\beta F_{12}+F_{21}\right|^{2} \\
& +\frac{\beta}{(1-s)(\beta-\alpha)^{2}}\left|\alpha F_{12}+F_{21}\right|^{2} .
\end{aligned}
$$

Proof. From (3.7), we obtain that

$$
r\left(s F_{11}^{\alpha, 1}+(1-s) F_{11}^{\beta, 1}\right)=F_{11}-s(1-r) c_{2}-(1-s)(1-r) c_{3} .
$$

Consequently, the problem is

$$
\min _{\left(S_{1}, S_{\gamma, \lambda}, c_{i}\right)} S_{1}+a_{\alpha} s\left(r S_{\alpha, 1}+(1-r) S_{\alpha, 0}\right)+a_{\beta}(1-s)\left(r S_{\beta, 1}+(1-r) S_{\beta, 0}\right)
$$

subject to

$(3.15)$

$$
\left\{\begin{aligned}
& \operatorname{det} F=\lambda\left(F_{11}-s(1-r) c_{2}-(1-s)(1-r) c_{3}\right)-S_{1} \\
&+\alpha s\left(r S_{\alpha, 1}+(1-r) S_{\alpha, 0}\right)+\beta(1-s)\left(r S_{\beta, 1}+(1-r) S_{\beta, 0}\right), \\
& S_{\alpha, 1} \geq c_{4}^{2}, \quad S_{\beta, 1} \geq c_{5}^{2}, \quad S_{\alpha, 0} \geq f_{4}^{2}\left(c_{4}\right), \quad S_{\beta, 0} \geq f_{5}^{2}\left(c_{5}\right), \quad S_{1} \geq\left(F_{11}\right)^{2} .
\end{aligned}\right.
$$

Copyright (c) by SIAM. Unauthorized reproduction of this article is prohibited. 
Since $a_{\alpha}$ and $a_{\beta}$ are positive, the minimum is obtained when the equalities hold in $(3.15)_{2}$ with a suitable choice of the constant $c_{2}$ and $c_{3}$ in $(3.15)_{1}$. Therefore, the minimum is

$$
\left|F_{11}^{2}\right|+a_{\alpha} s\left(r c_{4}^{2}+(1-r) f_{4}^{2}\left(c_{4}\right)\right)+a_{\beta}(1-s)\left(r c_{5}^{2}+(1-r) f_{5}^{2}\left(c_{5}\right)\right) .
$$

The minimization of $\left(r c_{4}^{2}+(1-r) f_{4}^{2}\left(c_{4}\right)\right)$ with respect to $c_{4}$ leads to

$$
c_{4}=\frac{1}{s(\beta-\alpha)}\left(\beta F_{12}+F_{21}\right)=F_{12}^{\alpha, 1}
$$

and then

$$
\left(r c_{4}^{2}+(1-r) f_{4}^{2}\left(c_{4}\right)\right)=\left(\frac{1}{s(\beta-\alpha)}\left(\beta F_{12}+F_{21}\right)\right)^{2}=c_{4}^{2}=S_{\alpha, 1} .
$$

Similarly, we obtain

$$
c_{5}=-\frac{1}{(1-s)(\beta-\alpha)}\left(\alpha F_{12}+F_{21}\right)=F_{12}^{\beta, 1} .
$$

Then, writing $\operatorname{det} F=F_{11} F_{22}-F_{12} F_{21}=-F_{11}^{2}+\lambda r F_{11}-F_{12} F_{21}$ from (3.6), the relation $(3.15)_{1}$ becomes

$$
\begin{gathered}
\lambda r F_{11}-F_{12} F_{21}=\lambda\left(F_{11}-s(1-r) c_{2}-(1-s)(1-r) c_{3}\right)+\frac{\alpha}{s(\beta-\alpha)^{2}}\left|\beta F_{12}+F_{21}\right|^{2} \\
+\frac{\beta}{(1-s)(\beta-\alpha)^{2}}\left|\alpha F_{12}+F_{21}\right|^{2}
\end{gathered}
$$

and implies the equality $\lambda(1-r) F_{11}=\lambda(1-r)\left(s c_{2}+(1-s) c_{3}\right)$, and then $\left(s c_{2}+\right.$ $\left.(1-s) c_{3}\right)=F_{11}$. This leads to the expression of $C P W$. Moreover, note that since $c_{2}=F_{11}^{\alpha, 0}$ and $c_{3}=F_{11}^{\beta, 0}$, the relation $F_{11}=s F_{11}^{\alpha, 0}+(1-s) F_{11}^{\beta, 0}$ implies

$$
F_{11}^{\alpha, 0}=F_{11}^{\beta, 0}=F_{11}
$$

and then, from $(3.15)_{2}$,

$$
F_{11}^{\alpha, 1}=F_{11}^{\beta, 1}=F_{11}
$$

Remark 3.3. From (3.6), $-\operatorname{det} F-\left|F_{11}\right|^{2}+\lambda r F_{11}$ is simply $F_{12} F_{21}$ and

$$
\begin{aligned}
\psi(F, s, r) & =F_{12} F_{21}+\frac{\alpha}{s(\beta-\alpha)^{2}}\left|\beta F_{12}+F_{21}\right|^{2}+\frac{\beta}{(1-s)(\beta-\alpha)^{2}}\left|\alpha F_{12}+F_{21}\right|^{2} \\
= & \frac{1}{s(1-s)(\beta-\alpha)^{2}}\left[F_{21}+F_{12}(\alpha s+\beta(1-s))\right]\left[\alpha \beta F_{12}+F_{21}(\alpha(1-s)+\beta s)\right]
\end{aligned}
$$

does not depend explicitly on $r$.

The polyconvexification $C P W$ gives a lower bound of the constrained quasi convexification. In the next section, we prove that this bound is in fact attained.

Copyright (c) by SIAM. Unauthorized reproduction of this article is prohibited. 


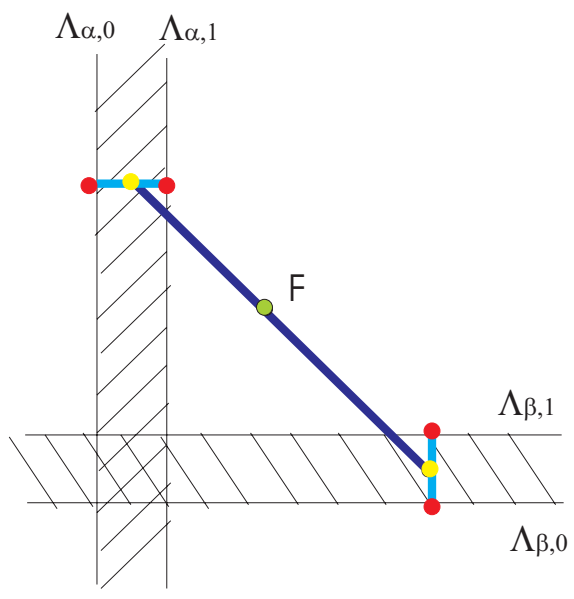

FIG. 3.1. Geometrical decomposition of F.

3.2. Upper bound: Searching laminates. In order to prove that the lower bound given by the polyconvexification is in fact the optimal value, we now search a measure $\nu$ in the class $\mathcal{A}_{\star}$ of laminates which recover it. Precisely, we exhibit a $\nu$ with the decomposition (3.3) and first moment $F$ which satisfies a rank one condition.

First, from the optimality conditions (3.5), (3.17), (3.21) and the strict convexity of the square function, we deduce that

$$
\nu^{(11)}=\delta_{F_{11}} \text { and } \nu_{\gamma, \lambda}^{(12)}=\delta_{F_{12}^{\gamma, \lambda}},
$$

and therefore

$$
\nu_{\gamma, \lambda}=\delta_{F^{\gamma, \lambda}} \quad \text { with } \gamma=\alpha, \beta, \lambda=0,1,
$$

where the matrices $F^{\gamma, \lambda}$ are

$$
F^{\gamma, 1}=\left(\begin{array}{cc}
F_{11} & y_{\gamma} \\
-\gamma y_{\gamma} & -F_{11}-\lambda
\end{array}\right), \quad F^{\gamma, 0}=\left(\begin{array}{cc}
F_{11} & y_{\gamma} \\
-\gamma y_{\gamma} & -F_{11}
\end{array}\right)
$$

with $\gamma=\alpha, \beta$ and

$$
y_{\alpha} \equiv \frac{1}{s(\beta-\alpha)}\left(\beta F_{12}+F_{21}\right), \quad y_{\beta} \equiv \frac{-1}{(1-s)(\beta-\alpha)}\left(\alpha F_{12}+F_{21}\right) .
$$

The unique possible measure $\nu$ which admits the decomposition (3.3) is then (geometrically; see Figure 3.1)

$$
\nu=s\left(r \delta_{F^{\alpha, 1}}+(1-r) \delta_{F^{\alpha, 0}}\right)+(1-s)\left(r \delta_{F^{\beta, 1}}+(1-r) \delta_{F^{\beta, 0}}\right) .
$$

Let us now check that $\nu$ is actually a laminate; i.e., we check that there is a rank one connection between the support of deltas. On the one hand, for $\gamma=\alpha, \beta$, the relation

$$
F^{\gamma, 1}-F^{\gamma, 0}=\left(\begin{array}{cc}
0 & 0 \\
0 & -\lambda
\end{array}\right)=b \otimes e_{2} \text { with } b=(0,-\lambda), e_{2}=(0,1)
$$


indicates that the direction of lamination of the set of damping has to be with normal $e_{2}$. On the other hand, the relation

$$
\begin{array}{r}
\left(r F^{\alpha, 1}+(1-r) F^{\alpha, 0}\right)-\left(r F^{\beta, 1}+(1-r) F^{\beta, 0}\right)=\left(\begin{array}{cc}
0 & y_{\alpha}-y_{\beta} \\
\beta y_{\beta}-\alpha y_{\alpha} & 0
\end{array}\right) \\
=\left(0, y_{\alpha}-y_{\beta}\right) \otimes e_{1}+\left(\alpha y_{\alpha}-\beta y_{\beta}, 0\right) \otimes e_{2}
\end{array}
$$

implies that $\nu$ is a laminate if and only if

$$
\operatorname{det}\left(\begin{array}{cc}
0 & y_{\alpha}-y_{\beta} \\
\beta y_{\beta}-\alpha y_{\alpha} & 0
\end{array}\right)=0 \quad \Longleftrightarrow \quad\left(y_{\alpha}-y_{\beta}\right)\left(\beta y_{\beta}-\alpha y_{\alpha}\right)=0 .
$$

Furthermore, from (3.22) and (3.23), we obtain that

$$
\psi(F, s, r)=s(1-s)\left(\alpha y_{\alpha}-\beta y_{\beta}\right)\left(y_{\alpha}-y_{\beta}\right) .
$$

Consequently, the above rank one condition is equivalent to $\psi(F, s, r)=0$, which is precisely the necessary condition for the polyconvexification to be finite (see Proposition 3.2). We then conclude that $\nu$ is a first order laminate, i.e., belongs to the class $\mathcal{A}_{\star}$. Then, we remark that the conditions $y_{\alpha}-y_{\beta}=0$ and $\alpha y_{\alpha}-\beta y_{\beta}=0$ are not compatible because they imply $y_{\alpha}=y_{\beta}=0$ and then $F_{12}=F_{21}=0$. We conclude that the direction of lamination of the $\alpha$ or $\beta$ material is $e_{2}=(0,1)$ if $y_{\alpha}-y_{\beta}=0$ or $e_{1}=(1,0)$ if $\beta y_{\beta}-\alpha y_{\alpha}=0$.

In conclusion, for the measure (3.24), the quasi convexification $C Q W$ defined by (2.9) coincides with $C P W$. Moreover, this provides an explicit expression of the full relaxation problem (2.11) stated in the following paragraph.

3.3. Well-posed full relaxation (RP). From Proposition 3.2 and by setting $\lambda=-d(x) U^{(1)}(t, x)=-d(x) u(t, x)$ and $F=\nabla U$ in (3.6), we obtain that the optimization problem

$$
(\mathrm{RP}) \min _{U, s, r} \hat{I}(U)=\int_{0}^{T} \int_{\Omega} C Q W(t, x, U(t, x), \nabla U(t, x), s(t, x), r(x)) d x d t
$$

subject to

$$
\left\{\begin{array}{l}
U=(u, v) \in\left(H^{1}([0, T] \times \Omega)\right)^{2}, \quad \psi(t, x, \nabla U(t, x), s(t, x), r(x))=0, \\
u_{t}+v_{x}=d(x) r(x) u(t, x) \quad \text { in } \quad \Omega \times(0, T), \\
U^{(1)}(0, x)=u_{0}(x), U_{t}^{(1)}(0, x)=u_{1}(x) \quad \text { in } \Omega, \\
U^{(1)}=0 \quad \text { in } \quad \partial \Omega \times[0, T], \\
0 \leq s(t, x) \leq 1, \quad \int_{\Omega} s(t, x) d x \leq L_{\alpha}|\Omega| \quad \forall t \in[0, T] \\
0 \leq r(x) \leq 1, \quad \int_{\Omega} r(x) d x \leq L_{d}|\Omega|
\end{array}\right.
$$

where

(3.28)

$\left.C Q W(U, F, s, r)=\left|F_{11}\right|^{2}+\frac{a_{\alpha}}{s(\beta-\alpha)^{2}}\left|\beta F_{12}+F_{21}\right|^{2}+\frac{a_{\beta}}{(1-s)(\beta-\alpha)^{2}} \mid \alpha F_{12}+F_{21}\right)\left.\right|^{2}$ 
and

$$
\begin{aligned}
\psi(F, s, r)=-\operatorname{det} F-\left|F_{11}\right|^{2}+\lambda r F_{11}+\frac{\alpha}{s(\beta-\alpha)^{2}}\left|\beta F_{12}+F_{21}\right|^{2} & \\
& +\frac{\beta}{(1-s)(\beta-\alpha)^{2}}\left|\alpha F_{12}+F_{21}\right|^{2}
\end{aligned}
$$

for any

$$
F=\left(\begin{array}{ll}
F_{11} & F_{12} \\
F_{21} & F_{22}
\end{array}\right), \quad s, r \in \mathbb{R},
$$

is a full well-posed relaxation of (VP) in the following sense.

THEOREM 3.4. The variational problem $(\mathrm{RP})$ is a relaxation of the initial optimization problem (VP) in the sense that

(a) the infima of both problems coincide;

(b) there are optimal solutions for the relaxed problem;

(c) these solutions codify (in the sense of the Young measures) the optimal microstructures of the original optimal design problem (see Theorem 3.5).

Moreover, we can compute explicitly optimal microstructures, as follows.

THEOREM 3.5. Optimal Young measures leading to the relaxed formulation are always first order laminates, which can be given in a completely explicit form:

- for the damping case the optimal microstructures are

$$
r(x) \delta_{1}+(1-r(x)) \delta_{0}
$$

with normal direction of lamination $e_{2}=(0,1)$;

- for the material case, the optimal microstructures are always

$$
s(x, t) \delta_{\alpha}+(1-s(x, t)) \delta_{\beta}
$$

with normal direction of lamination $e_{2}=(0,1)$ (if $y_{\alpha}-y_{\beta}=0$ ) or $e_{1}=(1,0)$ (if $\alpha y_{\alpha}-\beta y_{\beta}=0$ ), depending on each point.

Remark 3.6.

- The direction of lamination of the set of damping equal to $e_{2}=(0,1)$ is in full agreement with the time independence of the subset $\omega_{2}$, support of the dissipative term.

- It is interesting to note the influence of the damping term $\mathcal{X}_{\omega_{2}} d(x) u_{t}$ on the order of the laminates associated with the optimal Young measure. Without this damping term (i.e., when $\omega_{2}=\emptyset$ ), the analysis of the relaxation of $(\mathrm{P})$ (see [20]) reveals that the constrained quasi convexification is recovered by either first- or second order laminates, obtained when $\psi(\nabla U, s) \leq 0$ and $\psi(\nabla U, s)>0$, respectively. Here, even for arbitrarily small positive value of $\|d\|_{L^{\infty}(\Omega)}$ or $\left|\omega_{2}\right|$, the optimal laminates are always of first order, obtained on the set $\psi(\nabla U, s)=0$. This clearly highlights the smoother effect of this term.

4. Interpretation of the relaxed problem (RP) in terms of $u$. The quasiconvexified density depends on the gradient of $U$, verifies pointwise constraints, and may take the value $+\infty$ abruptly. For these reasons, the numerical approximation of the problem (RP) is not standard and is a priori tricky. In this section, taking advantage of the compatibility conditions, we analyze more deeply the relaxed formulation $(\mathrm{RP})$ and eliminate the auxiliary variable $v=U^{(2)}$ introduced in section 2. 
From the relation (3.26), the set $\{F ; \psi(F, s)=0\}$ is decomposed into two disjoint sets, $\left\{F ; y_{\alpha}-y_{\beta}=0\right\}$ and $\left\{F ; \alpha y_{\alpha}-\beta y_{\beta}=0\right\}$. Then, noticing that

$$
\left\{\begin{array}{l}
y_{\alpha}-y_{\beta}=0 \Longleftrightarrow F_{21}+F_{12}(\alpha s+\beta(1-s))=0, \\
\alpha y_{\alpha}-\beta y_{\beta}=0 \Longleftrightarrow F_{21}+F_{12} \frac{1}{\alpha^{-1} s+\beta^{-1}(1-s)}=0,
\end{array}\right.
$$

we may eliminate the variable $F_{21}$ (i.e., $v_{t}$ ) and write the quasi-convexified in terms of $F_{11}$ and $F_{12}$ only, as follows:

$$
C Q W(U, F, s, r)= \begin{cases}\left|F_{11}\right|^{2}+\left(a_{\alpha} s+a_{\beta}(1-s)\right)\left|F_{12}^{2}\right| & \text { if } y_{\alpha}-y_{\beta}=0 \\ \left|F_{11}\right|^{2}+\frac{a_{\alpha} \beta^{2} s+a_{\beta} \alpha^{2}(1-s)}{(\alpha(1-s)+\beta s)^{2}}\left|F_{12}^{2}\right| & \text { if } \alpha y_{\alpha}-\beta y_{\beta}=0 \\ +\infty & \text { else. }\end{cases}
$$

We can now invoke the following lemma (we refer to [12] for the proof).

Lemma 4.1. For all $s \in(0,1)$ and $0<\alpha<\beta$, we have

$$
\begin{aligned}
& \frac{a_{\beta}}{a_{\alpha}} \leq \frac{2 \beta}{\alpha+\beta} \Longrightarrow a_{\alpha} s+a_{\beta}(1-s) \leq \frac{a_{\alpha} \beta^{2} s+a_{\beta} \alpha^{2}(1-s)}{(\alpha(1-s)+\beta s)^{2}}, \\
& \frac{a_{\beta}}{a_{\alpha}} \geq \frac{\alpha+\beta}{2 \alpha} \Longrightarrow a_{\alpha} s+a_{\beta}(1-s) \geq \frac{a_{\alpha} \beta^{2} s+a_{\beta} \alpha^{2}(1-s)}{(\alpha(1-s)+\beta s)^{2}} .
\end{aligned}
$$

We are thus led to introducing the following problem:

$$
(\widetilde{\mathrm{RP}}): \inf _{s, r} \tilde{I}(s, r)=\int_{0}^{T} \int_{\Omega}\left(u_{t}(t, x)^{2}+G(s) u_{x}(t, x)^{2}\right) d x d t
$$

subject to

$$
\begin{cases}u_{t t}-\nabla_{x}\left(H(s) u_{x}\right)+d(x) r(x) u_{t}=0 & \text { in } \quad(0, T) \times \Omega, \\ u=0 & \text { on } \quad(0, T) \times \partial \Omega, \\ u(0, x)=u_{0}(x), u_{t}(0, x)=u_{1}(x) & \text { in } \Omega, \\ 0 \leq s(t, x) \leq 1, \quad \int_{\Omega} s(t, x) d x \leq L_{\alpha}|\Omega| & \text { in }[0, T] \times \Omega, \\ 0 \leq r(x) \leq 1, \quad \int_{\Omega} r(x) d x \leq L_{d}|\Omega| & \text { in } \Omega,\end{cases}
$$

where

$$
G(s)=a_{\alpha} s+a_{\beta}(1-s), \quad H(s)=\alpha s+\beta(1-s) \quad \text { if } \quad \frac{a_{\beta}}{a_{\alpha}} \leq \frac{2 \beta}{\alpha+\beta},
$$

and

$$
G(s)=\frac{a_{\alpha} \beta^{2} s+a_{\beta} \alpha^{2}(1-s)}{(\alpha(1-s)+\beta s)^{2}}, \quad H(s)=\frac{1}{\alpha^{-1} s+\beta^{-1}(1-s)} \quad \text { if } \quad \frac{a_{\beta}}{a_{\alpha}} \geq \frac{\alpha+\beta}{2 \alpha} .
$$

We assume henceforth that the positive functions $a_{\alpha}$ and $a_{\beta}$ fulfill, for all $x \in \Omega$, either the property $a_{\beta} / a_{\alpha} \leq 2 \beta /(\alpha+\beta)$ or $a_{\beta} / a_{\alpha} \geq(\alpha+\beta) / 2 \alpha$. 
Problem ( $\widetilde{\mathrm{RP}})$ with (4.6) (resp., (4.7)) is obtained from (RP) assuming that $C Q W$ is given by $(4.2)_{1}$ (resp., $(4.2)_{2}$ ), then putting $F=\nabla U$ and $\lambda=-d(x) u(t, x)$, and finally by eliminating the auxiliary variable $v$. Note that in the first case, $H$ is the arithmetic mean of $(\alpha, \beta)$, while in the second case, $H$ is the harmonic mean.

Moreover, one cannot affirm, a priori, that problem $(\widetilde{\mathrm{RP}})$ is equivalent to $(\mathrm{RP})$ because the pair $U=(u, v)$ which solves (RP) does not necessarily fulfill for all $(t, x) \in(0, T) \times \Omega$ the relation $v_{t}+u_{x}(\alpha s+\beta(1-s))=0$ (i.e., $y_{\alpha}-y_{\beta}=0$; see (4.1) with $F=\nabla U)$ or for all $(t, x)$ the relation $v_{t}+u_{x}\left(\alpha^{-1} s+\beta^{-1}(1-s)\right)^{-1}=0$ (i.e., $\left.\alpha y_{\alpha}-\beta y_{\beta}=0\right)$. However, we may conjecture this equivalence thanks to the following property.

LEMMA 4.2. The equality $\inf (\widetilde{\mathrm{RP}})=\min (\mathrm{RP})$ holds.

Proof. Let us consider the first case in Lemma 4.1, i.e., $a_{\alpha} / a_{\beta} \leq 2 \beta /(\alpha+\beta)$, leading to the arithmetic situation (4.6). In this case, $(\widetilde{\mathrm{RP}})$ is simply derived from (VP) by replacing the set of characteristic functions $\mathcal{X}_{\omega_{1}} \in L^{\infty}((0, T) \times \Omega,\{0,1\})$ by the larger set of density functions $s \in L^{\infty}((0, T) \times \Omega,(0,1))$. Therefore $\inf (\widetilde{\mathrm{RP}}) \leq$ $\inf (\mathrm{VP})$, and the conclusion follows from $\min (\mathrm{RP})=\inf (\mathrm{VP})$ (see Theorem 2.2) and $\min (\mathrm{RP}) \leq \inf (\widetilde{\mathrm{RP}})$. In the harmonic situation, we obtain the result using the same arguments and Lemma 4.1.

We have transformed the problem $(\mathrm{RP})$ into the problem $(\widetilde{\mathrm{RP}})$, where the auxiliary variable $v$ does not occur anymore and is much easier to solve numerically. We observe, however, that, since $(\widetilde{\mathrm{RP}})$ is not convex, one cannot ensure the existence of solutions. The next section aims at investigating the numerical resolution of $(\widetilde{\mathrm{RP}})$.

5. Numerical analysis of the relaxed problem. We address in this section the numerical resolution of the problem $(\widetilde{R P})$ in the quadratic case for which $\left(a_{\alpha}, a_{\beta}\right)=(1,1)$ and in the compliance case for which $\left(a_{\alpha}, a_{\beta}\right)=(\alpha, \beta)$. We first describe an algorithm of minimization and then present some numerical experiments. In order to simplify the presentation, we replace the volume constraint inequalities $(4.5)_{4}$ and $(4.5)_{5}$ by constraint equalities.

5.1. Algorithm of minimization. We present the resolution of the relaxed problem $(\widetilde{\mathrm{RP}})$ using a gradient descent method. In this respect, we compute the first variation of the cost function with respect to $s$ and $r$.

For any $\eta \in \mathbb{R}^{+}, \eta \ll 1$, and any $s_{1} \in L^{\infty}((0, T) \times \Omega)$, we associate with the perturbation $s^{\eta}=s+\eta s_{1}$ of $s$ the derivative of $\widetilde{I}$ with respect to $s$ in the direction $s_{1}$ as follows:

$$
\frac{\partial \widetilde{I}(s, r)}{\partial s} \cdot s_{1}=\lim _{\eta \rightarrow 0} \frac{\widetilde{I}\left(s+\eta s_{1}, r\right)-\widetilde{I}(s, r)}{\eta} .
$$

Theorem 5.1. If $\left(u_{0}, u_{1}\right) \in\left(H^{2}(\Omega) \cap H_{0}^{1}(\Omega)\right) \times H_{0}^{1}(\Omega)$, then the first derivative of $\widetilde{I}$ with respect to $s$ in any direction $s_{1}$ exists and takes the form

$$
\frac{\partial \widetilde{I}(s, r)}{\partial s} \cdot s_{1}=\int_{0}^{T} \int_{\Omega}\left(G_{, s}(s) u_{x}^{2}+H_{, s}(s) u_{x} p_{x}\right) s_{1} d x d t,
$$

where $u$ is the solution of $(4.5)$ and $p$ is the solution in $C^{1}\left([0, T] ; H_{0}^{1}(\Omega)\right) \cap$ $C^{1}\left([0, T] ; L^{2}(\Omega)\right)$ of the adjoint problem

$$
\begin{cases}p_{t t}-\nabla_{x}\left(H(s) p_{x}\right)-d(x) r(x) p_{t}=u_{t t}+\nabla_{x}\left(G(s) u_{x}\right) & \text { in } \quad(0, T) \times \Omega \\ p=0 & \text { on }(0, T) \times \partial \Omega \\ p(T, x)=0, \quad p_{t}(T, x)=u_{t}(T, x) & \text { in } \Omega\end{cases}
$$


Similarly, the first derivative of $\widetilde{I}$ with respect to $r$ in any direction $r_{1} \in L^{\infty}(\Omega)$ is given by

$$
\frac{\partial \widetilde{I}(s, r)}{\partial r} \cdot r_{1}=\int_{\Omega} d(x) r_{1}(x) \int_{0}^{T} u_{t}(t, x) p(t, x) d t d x .
$$

Proof. We introduce the Lagrangian

$$
\mathcal{L}(s, \phi, \psi)=\int_{0}^{T} \int_{\Omega}\left(\phi_{t}^{2}+G(s) \phi_{x}^{2}\right) d x d t+\int_{0}^{T} \int_{\Omega}\left[\phi_{t t}-\nabla_{x}\left(H(s) \phi_{x}\right)+d(x) r \phi_{t}\right] \psi d x d t
$$

for any $s \in L^{\infty}((0, T) \times \Omega), \phi \in C\left([0, T] ; H^{2}(\Omega) \cap H_{0}^{1}(\Omega)\right) \cap C^{1}\left([0, T] ; H_{0}^{1}(\Omega)\right)$, and $\psi \in C\left([0, T] ; H_{0}^{1}(\Omega)\right) \cap C^{1}\left([0, T] ; L^{2}(\Omega)\right)$ and then write formally that

$$
\frac{d \mathcal{L}}{d s} \cdot s_{1}=\frac{\partial}{\partial s} \mathcal{L}(s, \phi, \psi) \cdot s_{1}+\left\langle\frac{\partial}{\partial \phi} \mathcal{L}(s, \phi, \psi), \frac{\partial \phi}{\partial s} \cdot s_{1}\right\rangle+\left\langle\frac{\partial}{\partial \psi} \mathcal{L}(s, \phi, \psi), \frac{\partial \psi}{\partial s} \cdot s_{1}\right\rangle .
$$

The first term is

$$
\frac{\partial}{\partial s} \mathcal{L}(s, \phi, \psi) \cdot s_{1}=\int_{0}^{T} \int_{\Omega}\left(G_{, s}(s) \phi_{x}^{2}+H_{, s}(s) \phi_{x} \psi_{x}\right) s_{1} d x d t
$$

for any $s, \phi, \psi$, whereas the third term is equal to zero if $\phi=u$ is the solution of (4.5). We then determine the solution $p$ so that, for all $\phi \in C\left([0, T] ; H^{2}(\Omega) \cap H_{0}^{1}(\Omega)\right) \cap$ $C^{1}\left([0, T] ; H_{0}^{1}(\Omega)\right)$, we have

$$
\left\langle\frac{\partial}{\partial \phi} \mathcal{L}(s, \phi, p), \frac{\partial \phi}{\partial s} \cdot s_{1}\right\rangle=0
$$

which leads to the formulation of the adjoint problem (5.2). Next, writing that $\widetilde{I}(s)=$ $\mathcal{L}(s, u, p)$, we obtain (5.1) from (5.4). The relation (5.3) is obtained in a similar way.

In order to take into account the volume constraint on $s$ and $r$, we introduce the Lagrange multipliers $\gamma_{s} \in L^{\infty}((0, T) ; \mathbb{R}), \gamma_{r} \in \mathbb{R}$ and the functional

$$
\widetilde{I}_{\gamma}(s, r)=\widetilde{I}(s, r)+\int_{0}^{T} \gamma_{s}(t) \int_{\Omega} s(t, x) d x d t+\gamma_{r} \int_{\Omega} r(x) d x .
$$

Using Theorem 5.1, we then obtain easily that the first derivatives of $\widetilde{I}_{\gamma}$ are

$$
\begin{aligned}
& \frac{\partial \widetilde{I}_{\gamma}(s, r)}{\partial s} \cdot s_{1}=\int_{0}^{T} \int_{\Omega}\left(G_{, s}(s) u_{x}^{2}+H_{, s}(s) u_{x} p_{x}\right) s_{1} d x d t+\int_{0}^{T} \gamma_{s}(t) \int_{\Omega} s_{1} d x d t, \\
& \frac{\partial \widetilde{I}_{\gamma}(s, r)}{\partial r} \cdot r_{1}=\int_{\Omega} d(x) r_{1}(x) \int_{0}^{T} u_{t} p d x d t+\gamma_{r} \int_{\Omega} r_{1}(x) d x
\end{aligned}
$$

which lets us define the following descent directions, respectively:

$$
s_{1}(t, x)=-\left(G_{, s}(s) u_{x}^{2}+H_{, s}(s) u_{x} p_{x}+\gamma_{s}(t)\right) \quad \forall(t, x) \in(0, T) \times \Omega,
$$

and

$$
r_{1}(t, x)=-\left(d(x) \int_{0}^{T} u_{t}(t, x) p(t, x) d t+\gamma_{r}\right) \quad \forall x \in \Omega
$$

Copyright $@$ by SIAM. Unauthorized reproduction of this article is prohibited. 
Consequently, for any function $\eta_{s} \in L^{\infty}\left(\Omega \times(0, T), \mathbb{R}^{+}\right)$with $\left\|\eta_{s}\right\|_{L^{\infty}((0, T) \times \Omega)}$ small enough, we have $\widetilde{I}_{\gamma}\left(s+\eta_{s} s_{1}, r\right) \leq \widetilde{I}_{\gamma}(s, r)$. The multiplier function $\gamma_{s}$ is then determined so that, for any function $\eta_{s} \in L^{\infty}\left((0, T) \times \Omega, \mathbb{R}^{+}\right)$, ||$s+\eta_{s} s_{1} \|_{L^{1}(\Omega)}=L_{\alpha}|\Omega|$ for all $t \in(0, T)$, leading to

$$
\gamma_{s}(t)=\frac{\left(\int_{\Omega} s(t, x) d x-L_{\alpha}|\Omega|\right)-\int_{\Omega} \eta_{s}(t, x)\left(G_{, s}(s) u_{x}^{2}+H_{, s}(s) u_{x} p_{x}\right) d x}{\int_{\Omega} \eta_{s}(t, x) d x} .
$$

Finally, the function $\eta_{s}$ is chosen so that $s+\eta s_{1} \in[0,1]$ for all $(t, x) \in(0, T) \times \Omega$. A simple and efficient choice consists of taking $\eta_{s}(t, x)=\varepsilon s(t, x)(1-s(t, x))$ for all $(t, x) \in(0, T) \times \Omega$ with $\varepsilon$ small and positive.

Similarly, the choice

$$
\gamma_{r}=\frac{\left(\int_{\Omega} r(x) d x-L_{d}|\Omega|\right)-\int_{\Omega} \eta_{r}(x) d(x) \int_{0}^{T} u_{t}(t, x) p(t, x) d t d x}{\int_{\Omega} \eta_{r}(x) d x}
$$

with $\eta_{r}(x)=\varepsilon r(x)(1-r(x))$ for all $x \in \Omega$ permits us to ensure the condition $\| r+$ $\eta_{r} r_{1} \|_{L^{1}(\Omega)}=L_{d}|\Omega|$.

The descent algorithm to solve numerically the relaxed problem $(\widetilde{\mathrm{RP}})$ may be structured as follows.

Let $\Omega \subset \mathbb{R},\left(u_{0}, u_{1}\right) \in\left(H^{2}(\Omega) \cap H_{0}^{1}(\Omega)\right) \times H_{0}^{1}(\Omega), L_{\alpha}, L_{d} \in(0,1), T>0,0<\alpha<$ $\beta, a_{\beta}, a_{\alpha} \in L^{\infty}\left((0, T) \times \Omega ; \mathbb{R}_{+}^{\star}\right)$, and $\varepsilon<1, \varepsilon_{1} \ll 1$ be given;

- Initialization of the densities $s^{0} \in L^{\infty}\left((0, T \times \Omega ;] 0,1[)\right.$ and $r^{0} \in L^{\infty}(\Omega ;] 0,1[)$;

- For $k \geq 0$, iteration until convergence (i.e., $\left|\widetilde{I}_{\gamma}\left(s^{k+1}, r^{k+1}\right)-\widetilde{I}_{\gamma}\left(s^{k}, r^{k}\right)\right| \leq$ $\left.\varepsilon_{1}\left|\widetilde{I}_{\gamma}\left(s^{0}, r^{0}\right)\right|\right)$ as follows:

- Compute the solution $u_{s^{k}, r^{k}}$ of (4.5) and then the solution $p_{s^{k}, r^{k}}$ of (5.2), both corresponding to $(s, r)=\left(s^{k}, r^{k}\right)$.

- Compute the descent direction $s_{1}^{k}$ defined by (5.5), where the multiplier $\gamma^{k}$ is defined by (5.7). Similarly, compute the descent direction $r_{1}^{k}$ defined by (5.6), where the multiplier $\gamma^{k}$ is defined by (5.8).

- Update the density $s^{k}$ in $(0, T) \times \Omega$ and the density $r^{k}$ in $\Omega$ :

$$
s^{k+1}=s^{k}+\varepsilon s^{k}\left(1-s^{k}\right) s_{1}^{k}, \quad r^{k+1}=r^{k}+\varepsilon r^{k}\left(1-r^{k}\right) r_{1}^{k}
$$

with $\varepsilon \in \mathbb{R}^{+}$small enough to ensure the decrease of the cost function, $s^{k+1} \in L^{\infty}((0, T) \times \Omega,[0,1])$ and $r^{k+1} \in L^{\infty}(\Omega,[0,1])$.

5.2. Numerical experiments. In this section, we present some numerical simulations for $\Omega=(0,1)$ in the quadratic case - $\left(a_{\alpha}, a_{\beta}\right)=(1,1)$ - and in the compliance case $-\left(a_{\alpha}, a_{\beta}\right)=(\alpha, \beta)$. Recalling the assumption $0<\alpha<\beta$, these two cases fall into the arithmetic (see (4.6)) and harmonic (4.7) cases, respectively. From a numerical viewpoint, we highlight that the numerical resolution of the descent algorithm is a priori delicate in the sense that the descent direction depends on the derivative of $u$ and $p$, both solutions of a wave equation with space and time coefficients only in $L^{\infty}\left((0, T) \times \Omega ; \mathbb{R}_{+}^{*}\right)$. To the knowledge of the authors, there does not exist any numerical analysis for this kind of equation. We use a $C^{0}$-finite element approximation for $u$ and $p$ with respect to $x$ and a finite difference centered approximation with respect to $t$. Moreover, we add a vanishing viscosity and dissipative term of the type $(\beta-\alpha) \epsilon^{2} \operatorname{div}\left(H(s) u_{x t t}\right)$ with $\epsilon$ of order $h$-the space discretization parameter. This term has the effect of regularizing the descent term (5.5) and leading to a convergent algorithm. Finally, this provides an implicit and unconditionally stable scheme, consistent with (4.5) and (5.2), and of order two in time and space. 

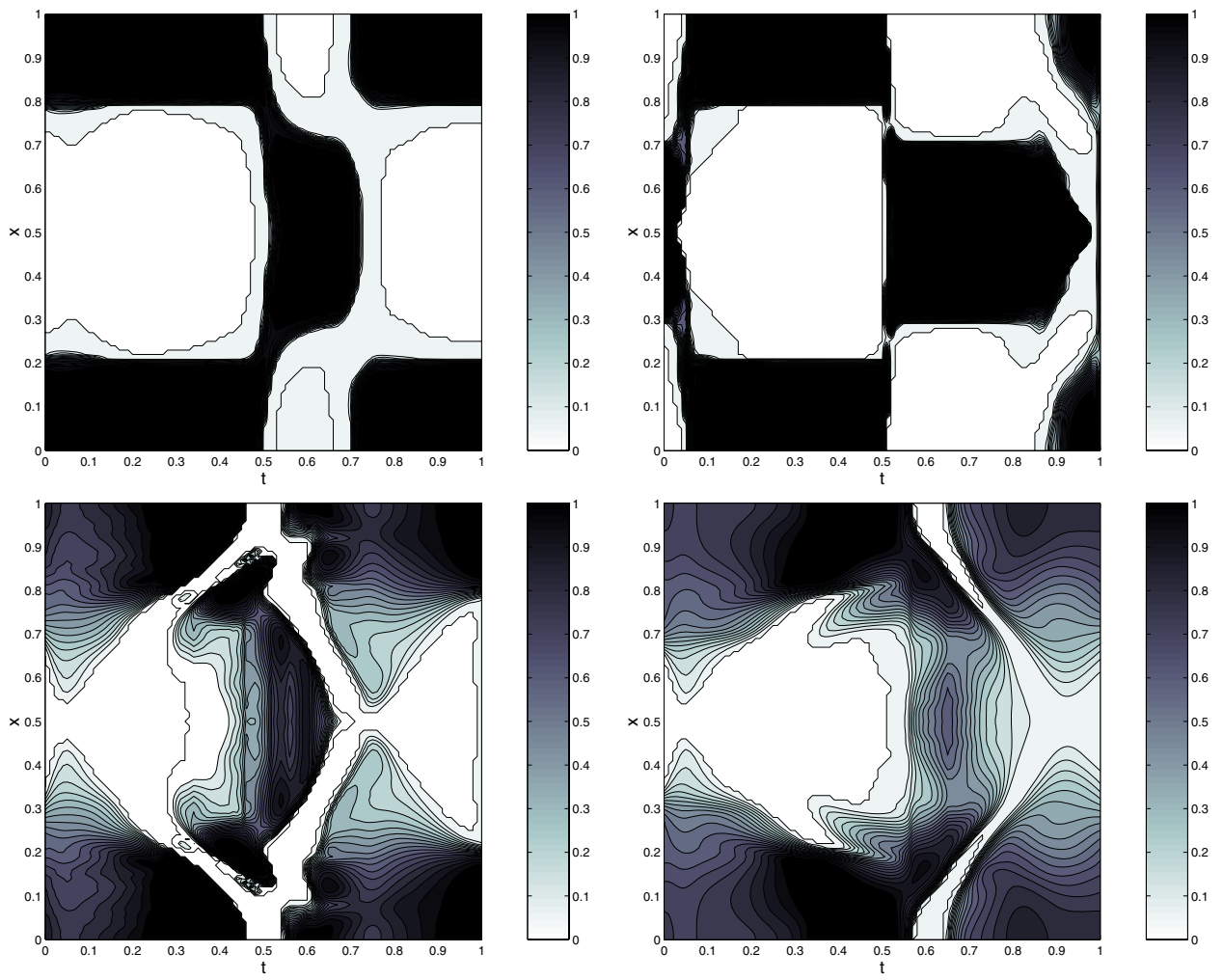

FIG. 5.1. $\left(a_{\alpha}, a_{\beta}\right)=(\alpha, \beta)$. Optimal density sim $^{\text {lim }}$ on $(0, T) \times \Omega$ for $(\alpha, \beta, d)=(1,1.1,1)$ (top left), $(\alpha, \beta, d)=(1,1.1,10)$ (top right), $(\alpha, \beta, d)=(1,4,1)$ (bottom left), and $(\alpha, \beta, d)=(1,4,10)$ (bottom right).

In what follows, we treat the following simple and smooth initial conditions on $\Omega=(0,1)$ :

$$
u_{0}(x)=\sin (\pi x), \quad u_{1}(x)=0,
$$

and $\alpha=1$. Results are obtained with $h=\Delta t=10^{-2}(\Delta t$ designates the time discretization parameter), $\varepsilon_{1}=10^{-5}, L_{\alpha}=2 / 5, L_{d}=1 / 5, T=1, s^{0}(t, x)=L_{\alpha}$ on $[0, T] \times \Omega, r^{0}(x)=L_{d}$ on $\Omega$, and $\varepsilon=10^{-2}$ (see the algorithm).

We highlight that the gradient algorithm may lead to local minima of $\widetilde{I}$ with respect to $s$ and $r$. For this reason, we consider constant initial density $s^{0}$ and $r^{0}$ as indicated above, which does not privilege any location for $\omega_{1}$ and $\omega_{2}$.

We discuss the result obtained with respect to the value of $\beta$ and of the damping function $d(x)=d \mathcal{X}_{\Omega}$ assumed constant in $\Omega$ : precisely, for $(\beta, d)=(1.1,1),(\beta, d)=$ $(1.1,10),(\beta, d)=(4,1)$, and $(\beta, d)=(4,10)$.

5.2.1. The compliance case- $\left(a_{\alpha}, a_{\beta}\right)=(\alpha, \beta)$. The compliance choice is the most usual one, because the corresponding cost function $I$ (see (1.3)) coincides with the energy of the vibrating membrane described by system (1.1). This case falls into the harmonic situation (4.7), $G(s)=H(s)=\left(\alpha^{-1} s+\beta^{-1}(1-s)\right)^{-1}$, and we get easily that $G_{, s}(s)=(\alpha-\beta) G^{2}(s) /(\alpha \beta)$. We present some results obtained with the following data: Figures 5.1 and 5.2 depict the iso-values of the optimal density 

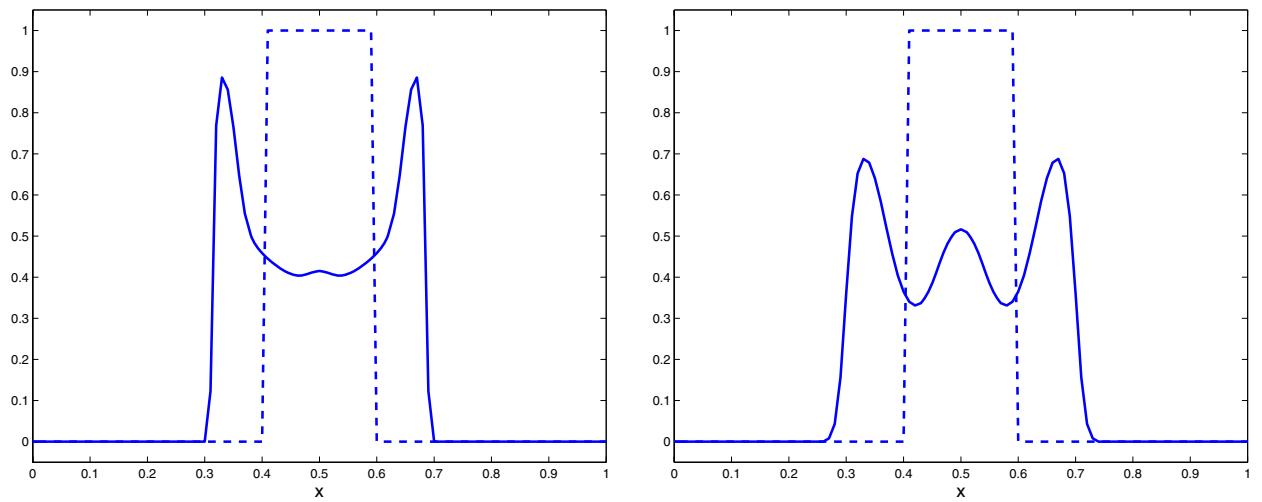

FIG. 5.2. $\left(a_{\alpha}, a_{\beta}\right)=(\alpha, \beta)$. Solid line: Optimal density $r^{l i m}$ for $(\alpha, \beta, d)=(1,1.1,10)$ (left) and $(\alpha, \beta, d)=(1,4,10)$ (right). Dashed line: Optimal density $r^{\text {lim }}=\mathcal{X}_{[0.4,0.6]}$ for $(\alpha, \beta, d)=(1,1.1,1)$ and $(\alpha, \beta, d)=(1,4,1)$.

$s^{\text {lim }}$ and $r^{\text {lim }}$, respectively (obtained at the convergence of the descent algorithm). In agreement with [20] (case $\omega_{2}=\emptyset$ ) and [22] (case $\omega_{1}=\emptyset$ ), results depend qualitatively on the gap $\beta-\alpha$ and $d$. When $\beta-\alpha$ and $d$ are small enough (function of the data of the problem), here $(\alpha, \beta, d)=(1,1.1,1)$, we observe that the optimal densities are characteristic functions. In this case, problem $(\widetilde{\mathrm{RP}})$ coincides with the original problem $(\mathrm{P})$ (we check that when $s^{l i m} \in L^{\infty}((0, T) \times \Omega,\{0,1\})$, i.e., $s^{\text {lim }}=\mathcal{X}_{\omega_{1}}$, then $\left.H\left(s^{l i m}\right)=\alpha s^{l i m}+\beta\left(1-s^{\lim }\right)=\alpha \mathcal{X}_{\omega_{1}}+\beta\left(1-\mathcal{X}_{\omega_{1}}\right)\right)$. The original problem is therefore well posed in the class of characteristic function: $\mathcal{X}_{\omega_{1}}=s^{\lim } \in L^{\infty}((0, T) \times \Omega ;\{0,1\})$ and $\mathcal{X}_{\omega_{2}}=r^{l i m} \in L^{\infty}(\Omega ;\{0,1\})$.

Precisely, $r^{l i m}=\mathcal{X}_{\left[1 / 2-L_{d} / 2,1 / 2+L_{d} / 2\right]}=\mathcal{X}_{[0.4,0.6]}$, and the optimal position for the damping zone is - as expected according to the symmetry of $u_{0}$ - the centered one: $\omega_{2}=[0.4,0.6]$. Moreover, the optimal distribution of $(\alpha, \beta)$-material is time dependent (see Figure 5.1(top left)), and we observe that the weaker material $\alpha$ (black zone on the figure) is located, for each time $t$, on the point $(x, t)$ where the amplitude of $u(x, t)$ is the lowest: on the extremities of $\Omega$ at time $t=0$, and on the middle at time $t \approx 0.5$.

If now we consider a larger gap $\beta-\alpha$, for instance $(\alpha, \beta, d)=(1,4,1)$, the limit density $s^{\text {lim }}$ is no longer a characteristic function and takes values in $(0,1)$, highlighting microstructure (Figure 5.1(bottom left)). This suggests that the initial problem $(\mathrm{P})$ is not well posed in the class of characteristic functions and does not coincide with the relaxed problem $(\widetilde{\mathrm{RP}})$. This also fully justifies the search and introduction of a relaxed well-posed formulation. We observe also that this gap is not enough larger to influence the density $r^{l i m}$ : we still have $r^{l i m}=\mathcal{X}_{[0.4,0.6]}$.

Similarly, when we increase the value of the damping function $d$ (and therefore the dissipation of the system), the limit density $r^{\text {lim }}$ is no longer a characteristic function (see Figure 5.2 for $(\alpha, \beta, d)=(1,1.1,10)$ (left) and $(\alpha, \beta, d)=(1,4,10)$ (right)) but remains symmetric with respect to $x=1 / 2$. The optimal domain is no longer the centered position but an infinite union of disjoint intervals (see section 5.2.3). This damping term with $d=10$ changes significantly the dynamic of $u$ and perturbs the optimal dynamical distribution of $(\alpha, \beta)$-material (see Figure 5.1(right)). For $(\alpha, \beta, d)=(1,1.1,10)$, the function $s^{l i m}$ remains a characteristic function.

Finally, we plot the integrand of the cost function $\widetilde{I}$, i.e., the energy $E(t) \equiv$ $\int_{\Omega}\left(\left|u_{t}\right|^{2}+G\left(s^{\text {lim }}\right)\left|u_{x}\right|^{2}\right) d x$ with respect to time (Figure 5.3). Although the system is 


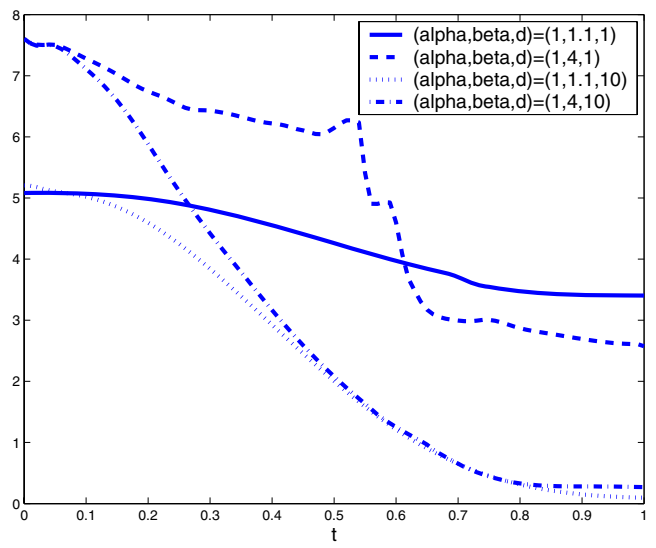

FIG. 5.3. $\left(a_{\alpha}, a_{\beta}\right)=(\alpha, \beta)$. Evolution of $\int_{\Omega}\left(\left|u_{t}\right|^{2}+G\left(s^{l i m}\right)\left|u_{x}\right|^{2}\right) d x$ versus $t \in[0, T]$.

not necessarily dissipative when $\omega_{2}=\emptyset$-we have the relation

$$
\begin{aligned}
\frac{d E(t)}{d t} & =2 \int_{\Omega} H_{t}(s) u_{x}^{2} d x-2 \int_{\Omega} d(x) r(x) u_{t}^{2} d x \\
& =2 \alpha \beta(\alpha-\beta) \int_{\Omega} \frac{s_{t}}{(\alpha(1-s)+\beta s)^{2}} u_{x}^{2} d x-2 \int_{\Omega} d(x) r(x) u_{t}^{2} d x
\end{aligned}
$$

-we observe that the optimal $(\alpha, \beta)$-distribution leads to a dissipative system and that the dissipation is monotonous with respect to $(\beta-\alpha)$.

5.2.2. The quadratic case- $\left(a_{\alpha}, a_{\beta}\right)=(1,1)$. This case falls in the arithmetic situation (see (4.6)), and the relaxed problem $(\widetilde{\mathrm{RP}})$ is then simply derived from the original one by replacing $\left(\mathcal{X}_{\omega_{1}}, \mathcal{X}_{\omega_{2}}\right)$ by $(s, r)$.

Once again, the optimal distribution of $(\alpha, \beta)$ and damping material strongly depends on the gap of the coefficients. Moreover, the numerical results still suggest that the original problem is not well posed if these gaps exceed critical values depending on the data (see Figure 5.4). The main difference with respect to the compliance case is observed for $(\alpha, \beta, d)=(1,4,10)$ : it appears that the density $r^{l i m}$ is a characteristic function: $r^{l i m}=\mathcal{X}_{[0.4,0.6]}$ (see Figure 5.5). A greater value of $d$ (for instance, $d=15$ ) is necessary to obtain values in $(0,1)$. This phenomenon is due to the dissipative effect of the optimal $(\alpha, \beta)$-distribution and highlights the interaction between $s$ and $r$ (or equivalently between $\omega_{1}$ and $\left.\omega_{2}\right)$.

Contrary to the compliance case where the density varies somewhat smoothly (see Figure 5.1), we observe in the bottom two panels in Figure 5.4 some high oscillations of the optimal density $s$ with respect to both $t$ and $x$ (especially with $(\alpha, \beta, d)=$ $(1,4,10))$. Due to the nonconvexity of the functional $\tilde{I}(s, r)$ with respect to $s$ in the quadratic case, we recall that we do not know a priori whether the problem $(\widetilde{\mathrm{RP}})$ defined by $(4.4)$ is well posed: we can only ensure that $\inf (\widetilde{\mathrm{RP}})=\min (\mathrm{RP})$ (Lemma 4.2). The situation is different in the compliance case because $\tilde{I}$ is convex. Therefore, these oscillations may be related to the possible ill-posedness of $(\widetilde{\mathrm{RP}})$. These oscillations may also be caused, at least partially, by the numerical sensitivity of the approximation, as discussed above. Figure 5.6 depicts the evolution of the energy for the different values of $\alpha, \beta$, and $d$. 

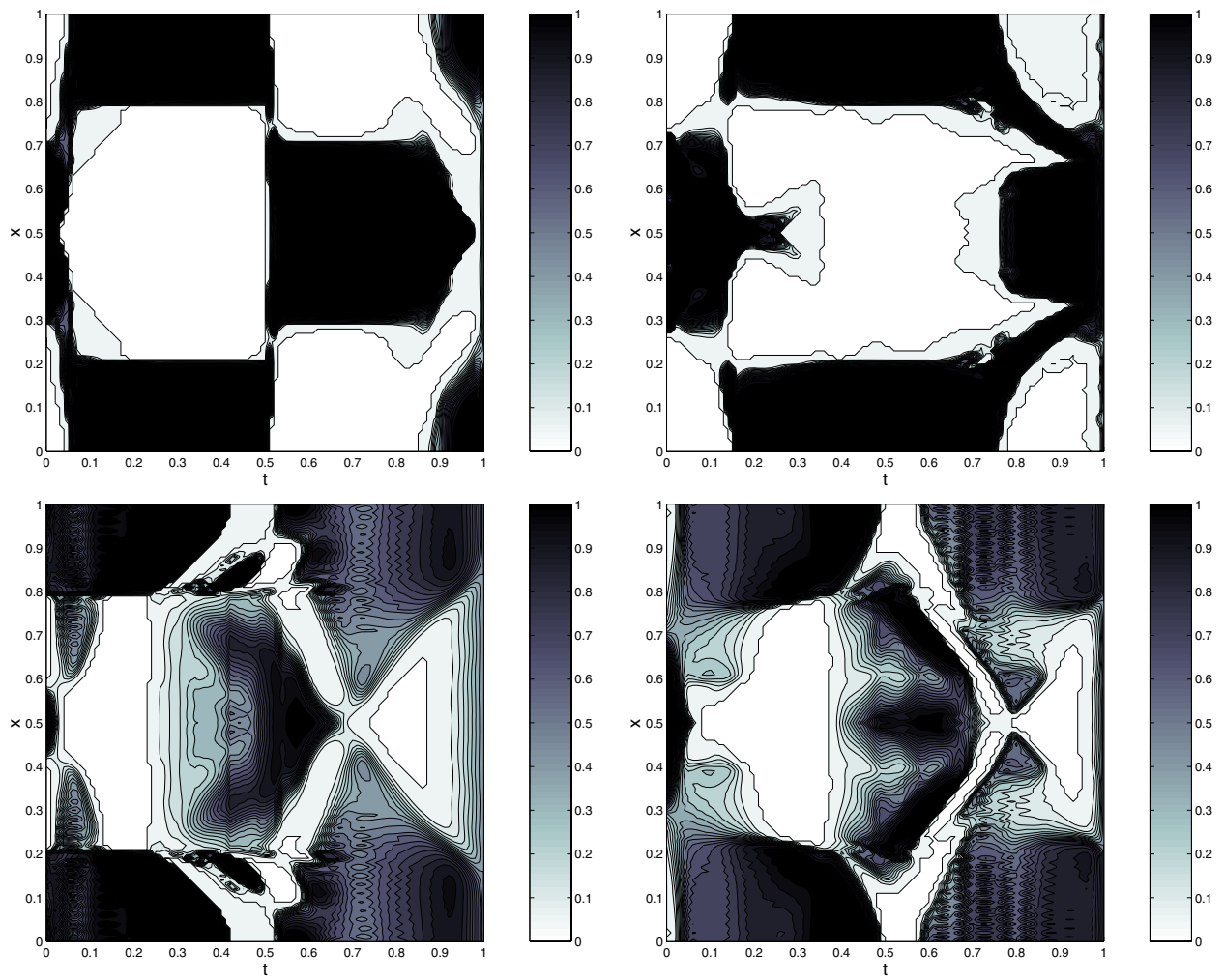

FIG. 5.4. $\left(a_{\alpha}, a_{\beta}\right)=(1,1)$. Optimal density sim $^{\text {lim }}(t, x)$ on $\Omega \times(0, T)$ for $(\alpha, \beta, d)=(1,1.1,1)$ (top left), $(\alpha, \beta, d)=(1,1.1,10)$ (top right), $(\alpha, \beta, d)=(1,4,1)$ (bottom left), and $(\alpha, \beta, d)=(1,4,10)$ (bottom right).

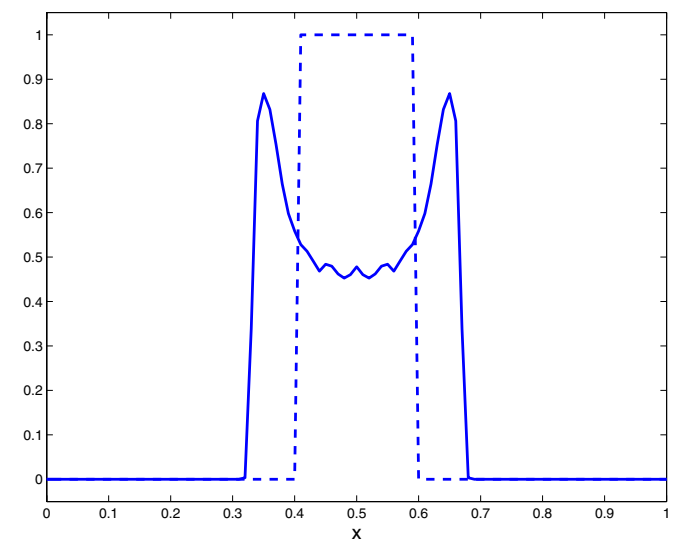

FIG. 5.5. $\left(a_{\alpha}, a_{\beta}\right)=(1,1)$. Solid line: Optimal density $r^{\text {lim }}$ for $(\alpha, \beta, d)=(1,1.1,10)$. Dashed line: Optimal density $r^{\text {lim }}=\mathcal{X}_{[0.4,0.6]}$ for $(\alpha, \beta, d)=(1,1.1,1),(\alpha, \beta, d)=(1,4,1)$, and $(\alpha, \beta, d)=$ $(1,4,10)$.

5.2.3. Extraction of a minimizing sequence $\left(\mathcal{X}_{\omega_{1}^{k}}, \mathcal{X}_{\omega_{2}^{k}}\right)$ from the optimal density $\left(s^{l i m}, r^{l i m}\right)$. Once we have the optimal microstructure of the $(\alpha, \beta)$ material and damping material codified by the optimal density $s$ and $r$, it remains 


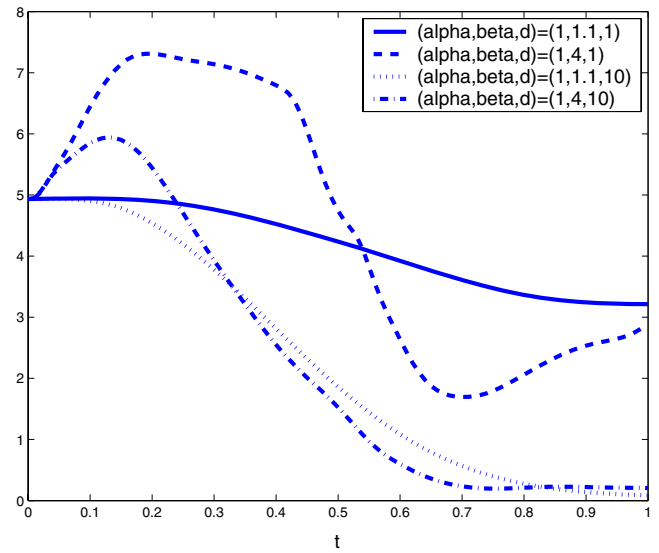

FIG. 5.6. $\left(a_{\alpha}, a_{\beta}\right)=(1,1)$. Evolution of $\int_{\Omega}\left(\left|u_{t}\right|^{2}+G\left(s^{l i m}\right)\left|u_{x}\right|^{2}\right) d x$ versus $t \in[0, T]$.

(from a practical viewpoint) to extract from $\left(s^{\text {lim }}, r^{l i m}\right)$, a sequence of characteristic functions $\left(\mathcal{X}_{\omega_{1}^{k}}, \mathcal{X}_{\omega_{2}^{k}}\right)$ such that $\lim _{k \rightarrow \infty} \widetilde{I}\left(\mathcal{X}_{\omega_{1}^{k}}, \mathcal{X}_{\omega_{2}^{k}}\right)=\widetilde{I}\left(s^{l i m}, r^{l i m}\right)$.

Recalling that $r^{\lim }(x)$ is the volume fraction of the damping material at point $x$, we proceed as follows. Let us decompose the interval $\Omega$ into $M>0$ nonempty subintervals such that $\Omega=\cup_{j=1, M}\left[x_{j}, x_{j+1}\right]$. Then, we associate with each interval $\left[x_{j}, x_{j+1}\right]$ the mean value $m_{j} \in[0,1]$ defined by

$$
m_{j}=\frac{1}{x_{j+1}-x_{j}} \int_{x_{j}}^{x_{j+1}} r^{\lim }(x) d x
$$

and the division into two parts

$$
\left[x_{j},\left(1-m_{j}\right) x_{j}+m_{j} x_{j+1}\right] \cup\left[\left(1-m_{j}\right) x_{j}+m_{j} x_{j+1}, x_{j+1}\right] .
$$

Finally, we introduce the function $r_{M}^{p e n}$ in $L^{\infty}(\Omega,\{0,1\})$ by

$$
r_{M}^{p e n}(x)=\sum_{j=1}^{M} \mathcal{X}_{\left[x_{j},\left(1-m_{j}\right) x_{j}+m_{j} x_{j+1}\right]}(x)
$$

We easily check that $\left\|r_{M}^{p e n}\right\|_{L^{1}(\Omega)}=\left\|r^{\text {lim }}\right\|_{L^{1}(\Omega)}$ for all $M>0$. The bivalued function $r_{M}^{p e n}$ takes more advantage of the information codified in the density $r^{l i m}$. Similarly, using that $s(t, x)$ is the volume fraction of the $\alpha$-material at point $(t, x)$, we associate with $s^{\lim }$ a sequence of bivalued functions $s_{N}^{p e n} \in L^{\infty}((0, T) \times \Omega,\{0,1\})$ (see [20]).

For $(\alpha, \beta, d)=(1,4,10)$ and $\left(a_{\alpha}, a_{\beta}\right)=(\alpha, \beta)$, Figure 5.7 represents the function $r_{M=30}^{p e n}$ associated with the density $r^{\text {lim }}$ of Figure 5.2(right). Similarly, Figure 5.8 represents the function $s_{N=30}^{p e n}$ associated with the optimal density $s^{\text {lim }}$ of Figure 5.1(bottom right). Finally, we report in Table 5.1 values of $\widetilde{I}\left(s_{N}^{p e n}, r_{M}^{p e n}\right)$ for several values of $N$ and $M$. For $M=N=40$, we obtain $\widetilde{I}\left(s_{40}^{p e n}, r_{40}^{p e n}\right) \approx 2.9803$ which is very near from the minimal value $I\left(s^{l i m}, r^{l i m}\right) \approx 2.9116$. These numerical results suggest the efficiency of this procedure to build optimal domains $\omega_{1}, \omega_{2}$ composed of a finite number of disjoints components and arbitrarily near the optimal distributions. 


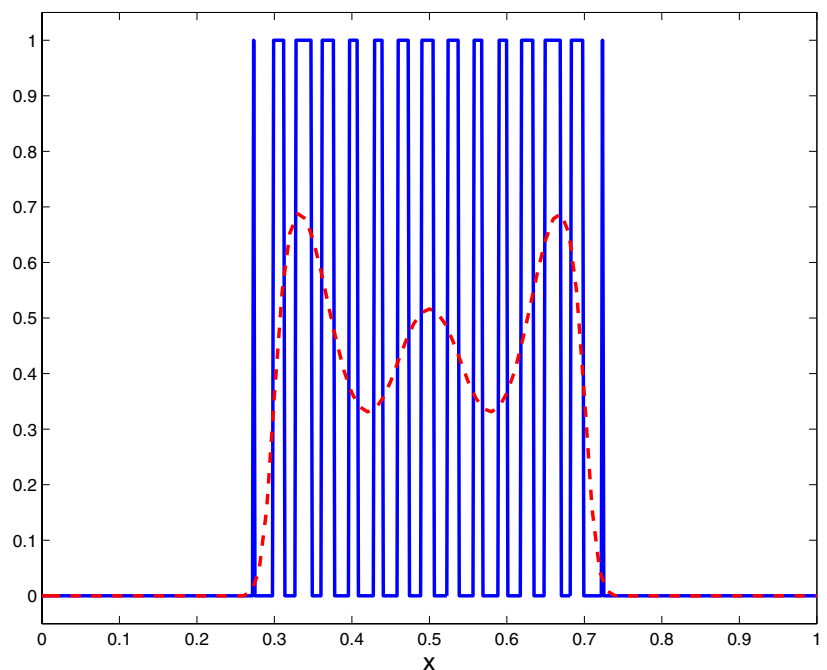

FIG. 5.7. $\left(a_{\alpha}, a_{\beta}\right)=(\alpha, \beta)$. Characteristic function associated with the optimal density $r^{\text {lim }}$ for $(\alpha, \beta, d)=(1,4,10)$. $\widetilde{I}\left(s^{l i m}, r^{l i m}\right) \approx 2.9116$. $\widetilde{I}\left(s^{l i m}, r_{M=30}^{\text {pen }}\right) \approx 3.0360$.

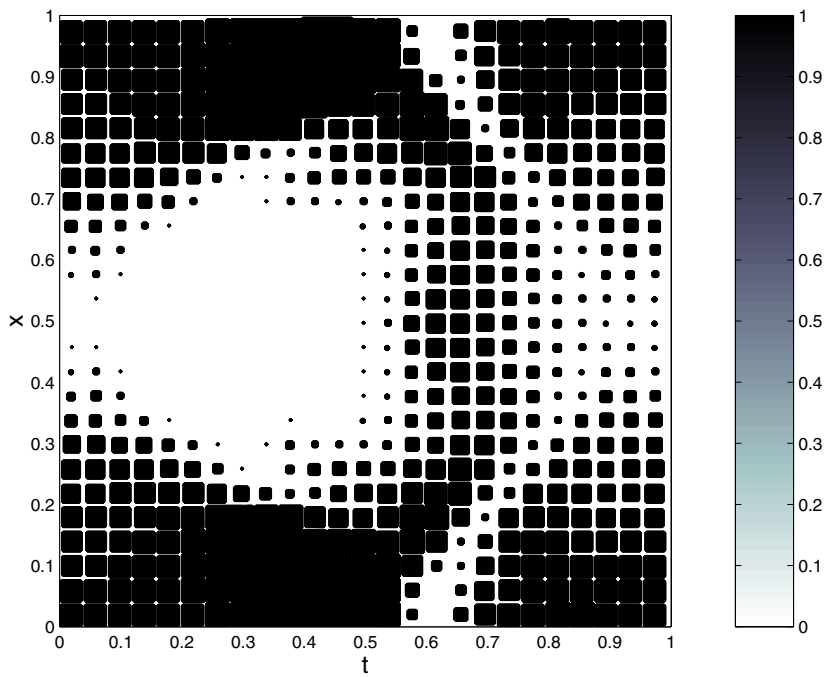

FIG. 5.8. $\left(a_{\alpha}, a_{\beta}\right)=(\alpha, \beta)$. Characteristic function associated with the optimal density $s^{\text {lim }}$ for $(\alpha, \beta, d)=(1,4,10)$. $\widetilde{I}\left(s^{l i m}, r^{l i m}\right) \approx 2.9116$. $\widetilde{I}\left(s_{N=30}^{\text {pen }}, r^{l i m}\right) \approx 3.0755$.

TABLE 5.1

$\left(a_{\alpha}, a_{\beta}\right)=(\alpha, \beta) . \quad(\alpha, \beta, d)=(1,4,10)$-Value of the cost function $\widetilde{I}\left(s_{N}^{p e n}, r_{M}^{p e n}\right)$ for $M, N \in$ $\{10,20,30,40\}$.

\begin{tabular}{c||cccc}
\hline$N \backslash M$ & 10 & 20 & 30 & 40 \\
\hline \hline 10 & 5.6181 & 5.2869 & 4.7629 & 4.4181 \\
20 & 5.0940 & 4.4721 & 4.0761 & 3.6712 \\
30 & 4.4910 & 3.8931 & 3.4612 & 3.1321 \\
40 & 4.2192 & 3.4821 & 3.0712 & 2.9803 \\
\hline
\end{tabular}


6. Concluding remarks and perspectives. We have analyzed the response of a 1-D damped string with respect to the spatio-temporal distribution of its longitudinal stiffness. The relaxed formulation highlights the smoothing effect of the damping term on the optimal spatio-temporal layout. Moreover, the numerical experiments indicate the strong dependence of the optimal distribution on the initial data $\left(u_{0}, u_{1}\right)$. In order to get free of this dependence, it would be interesting to consider, for instance, an inf-sup problem of the form

$$
\inf _{\mathcal{X}_{\omega_{1}}, \mathcal{X}_{\omega_{2}}} \sup _{\left(u_{0}, u_{1}\right) \in H_{0}^{1}(\Omega) \times L^{2}(\Omega)} I\left(\mathcal{X}_{\omega_{1}}, \mathcal{X}_{\omega_{2}}, u_{0}, u_{1}\right),
$$

where $I$ designates the cost function (1.3). Another approach may consist of averaging the cost function over all initial data of unit energy (we refer to [10] in a similar context). Finally, at the numerical level, it seems important to investigate the numerical approximation of the fully relaxed problem (RP) and compare it with the simplified formulation $(\widetilde{\mathrm{RP}})$. These aspects will be addressed in the near future.

\section{REFERENCES}

[1] G. Allaire, Shape Optimization by the Homogenization Method, Springer, New York, 2002.

[2] E. Aranda And P. Pedregal, Constrained envelope for a general class of design problems, Discrete Contin. Dynam. Systems, Supplement (2003), pp. 30-41.

[3] J. C. Bellido And A. Donoso, On an Optimal Design Problem in Wave-Propagation, preprint 3-2007, Universidad Castilla-La-Mancha, Ciudad Real, Spain.

[4] M. P. Bendsøe And O. Sigmund, Topology Optimization: Theory, Methods, and Applications, Springer, Berlin, Heidelberg, New York, 2003.

[5] D. Bucur and G. Buttazzo, Variational Methods in Shape Optimization Problems, Progr. Nonlinear Differential Equations Appl. 65, Birkhäuser, Basel, 2005.

[6] M. Burger and S. J. Osher, A survey on level set methods for inverse problems and optimal design, European J. Appl. Math., 16 (2005), pp. 263-301.

[7] J. Cagnol and J-.P. Zolésio, Shape control in hyperbolic problems. Optimal control of partial differential equations, in Internat. Ser. Numer. Math. 133, Birkhäuser, Basel, 1999, pp. $77-88$.

[8] A. Chambolle and F. Santosa, Control of the wave equation by time-dependent coefficient, ESAIM Control Optim. Calc. Var., 8 (2002), pp. 375-392.

[9] S. J. Cox, Designing for optimal energy absorption II, The damped wave equation, in Internat. Ser. Numer. Math. 126, Birkhäuser, Basel, 1998, pp. 103-109.

[10] S. J. Cox, I. NAkić, A. Rittmann, ANd K. Veselić, Lyapunov optimization of a damped system, Systems Control Lett., 53 (2004), pp. 187-194.

[11] B. Dacorogna, Direct Method in the Calculus of Variations, Springer, New York, 1989.

[12] A. Donoso And P. Pedregal, Optimal design of 2-D conducting graded materials by minimizing quadratic functionals in the field, Struct. Multidisc. Optim., 30 (2005), pp. 360-367.

[13] F. Fahroo and K. Iто, Variational formulation of optimal damping designs, Contemp. Math., 209 (1997), pp. 95-114.

[14] V. Girault and P.-A. Raviart, Finite Element Methods for Navier-Stokes equations. Theory and Algorithms, Springer, New York, 1986.

[15] P. Hebrard and A. Henrot, Optimal shape and position of the actuators for the stabilization of a string, Systems Control Lett., 48 (2003), pp. 199-209.

[16] J.-L. Lions and E. Magenes, Problèmes aux limites non homogènes et applications, Dunod, Paris, 1968

[17] K. LuRIE, Control of the coefficients of linear hyperbolic equations via spatio-temporal composites, in Homogenization, V. Berdychevisky, V. Jikov, and G. Papanicolau, eds., World Scientific, Singapore, 1999, pp. 285-315.

[18] K. LuRIE, Some new advances in the theory of dynamic materials, J. Elasticity, 72 (2003), pp. 229-239.

[19] F. Maestre And P. Pedregal, Quasiconvexification in 3-D for a variational reformulation of an optimal design problem in conductivity, Nonlinear Anal., 64 (2006), pp. 1962-1976.

[20] F. Maestre, A. Münch, And P. Pedregal, Optimal design under the one-dimensional wave equation, Interfaces Free Bound., to appear.

Copyright $@$ by SIAM. Unauthorized reproduction of this article is prohibited. 
[21] A. Münch, P. Pedregal, And F. Periago, A variational approach to a shape design problem for the wave equation, C. R. Acad. Sci. Paris Sér. I, 343 (2006), pp. 371-376.

[22] A. Münch, P. Pedregal, and F. Periago, Optimal design of the damping set for the stabilization of the wave equation, J. Differential Equations, 231 (2006), pp. 331-358.

[23] A. Münch, P. Pedregal, and F. Periago, Optimal Internal Design Stabilization of the Linear System of Elasticity, preprint 2007/01, Université de Franche-Comté, Bensancon, France.

[24] F. Murat, Contre-exemples pour divers problèmes où le contrôle intervient dans les coefficients, Ann. Mat. Pura Appl. Ser., 4 (1977), pp. 49-68.

[25] P. Pedregal, Parametrized measures and variational principles, Birkhäuser, Berlin, 1997.

[26] P. Pedregal, Vector variational problems and applications to optimal design, ESAIM Control Optim. Calc. Var., 15 (2005), pp. 357-381.

[27] J. SoKOŁOWSKI AND A. ŻochowSKI, On the topological derivative in shape optimization, SIAM J. Control Optim., 37 (1999), pp. 1251-1272.

Copyright (C) by SIAM. Unauthorized reproduction of this article is prohibited. 